\section{Research Square}

\title{
Acetylation reprograms MITF target selectivity
}

Colin Goding ( $\boldsymbol{\nabla}$ colin.goding@ludwig.ox.ac.uk )

University of Oxford https://orcid.org/0000-0002-1614-3909

Pakavarin Louphrasitthiphol

University of Oxford https://orcid.org/0000-0001-6546-332X

Alessia Loffreda

Istituto Scientifico Ospedale San Raffaele

Vivian Pogenberg

University Medical Center Hamburg Eppendorf https://orcid.org/0000-0002-1021-6804

\section{Sarah Picaud}

University of Oxford

\section{Alex Schepsky}

Ludwig Institute for Cancer Research

\section{Hans Friedrichsen}

University of Oxford

\section{Zhiqiang Zeng}

MRC Human Genetics Unit

\section{Benjamin Thomas}

University of Oxford

\section{E Patton}

MRC human Genetics Unit, Edinburgh https://orcid.org/0000-0002-2570-0834

\section{Matthias Wilmanns}

European Molecular Biology Laboratory https://orcid.org/0000-0002-4643-5435

\section{Panagis Filippakopoulos}

University of Oxford

\section{Eirikur Steingrimsson}

University of Iceland

\section{Davide Mazza}

San Raffaele Scientific Institute

\section{Article}

Keywords: Acetylation, E-box, DNA-binding affinity, MITF, bHLH-LZ, Melanoma 
DOI: https://doi.org/10.21203/rs.3.rs-1170720/v1

License: (c) (i) This work is licensed under a Creative Commons Attribution 4.0 International License. Read Full License 


\section{Acetylation reprograms MITF target selectivity}

\section{Pakavarin Louphrasitthiphol ${ }^{1,2}$, Alessia Loffreda ${ }^{3}$, Vivian Pogenberg ${ }^{4,10}$, Sarah Picaud ${ }^{5}$, Alexander Schepsky ${ }^{1,6}$, Hans Friedrichsen ${ }^{1}$, Zhiqiang Zeng ${ }^{7}$, Benjamin Thomas ${ }^{8}$, \\ E. Elizabeth Patton ${ }^{7}$, Matthias Wilmanns ${ }^{4,9}$, Panagis Filippakopoulos ${ }^{5}$, Eiríkur Steingrímsson $^{6}$, Davide Mazza ${ }^{3}$ and Colin R Goding ${ }^{*}$}

${ }^{1}$ Ludwig Institute for Cancer Research, Nuffield Department of Clinical Medicine, University of Oxford, Headington, Oxford, OX3 7DQ, UK.

${ }^{2}$ Department of Gastrointestinal and Hepato-Biliary-Pancreatic Surgery, Faculty of Medicine, University of Tsukuba, 1-1-1 Tennodai, Tsukuba, Ibaraki, 305-8575, Japan

${ }^{3}$ Fondazione CEN, European Center for Nanomedicine, Milano, 20133, Italy

${ }^{4}$ EMBL Hamburg c/o DESY, Notkestraße 25a, 22607, Hamburg, Germany.

${ }^{5}$ Structural Genomics Consortium, Nuffield Department of Clinical Medicine, University of Oxford, Headington, Oxford, OX3 7DQ, UK.

${ }^{6}$ Department of Biochemistry and Molecular Biology, BioMedical Center, Faculty of Medicine, University of Iceland, Sturlugata 8, 101 Reykjavik, Iceland.

${ }^{7}$ MRC Institute of Genetics and Molecular Medicine, MRC Human Genetics Unit \& Edinburgh Cancer Research Centre, Crewe Road South, Edinburgh, EH4 2XR, UK

${ }^{8}$ Central Proteomics Facility, Sir William Dunn Pathology School, Oxford University, Oxford, OX1 3RE, UK

${ }^{9}$ University Hamburg Medical Centre Hamburg-Eppendorf, Martinistrasse 52, 20246 Hamburg, Germany

${ }^{10}$ Present address: Institute of Biochemistry and Signal Transduction, University Hamburg Medical Centre Hamburg-Eppendorf, Martinistrasse 52, 20246 Hamburg, Germany

Running Title: Acetylation drives MITF target selectivity

Key words: Acetylation; E-box; DNA-binding affinity; MITF; bHLH-LZ; Melanoma;

* Corresponding Author:

Colin R Goding:

E-mail: colin.goding@ludwig.ox.ac.uk;

Tel: +44 1865617540 
The ability of transcription factors to discriminate between different classes of binding sites associated with specific biological functions underpins effective gene regulation in development and homeostasis. How this is achieved is poorly understood. The microphthalmia-associated transcription factor MITF is a lineage-survival oncogene that plays a crucial role in melanocyte development and melanoma. MITF suppresses invasion, reprograms metabolism and promotes both proliferation and differentiation. How MITF distinguishes between differentiation and proliferation-associated targets is unknown. Here we show that compared to many transcription factors MITF exhibits a very long $(>100 \mathrm{~s})$ residence time which is reduced by p300/CBP-mediated MITF acetylation at K206. While K206 acetylation also decreases genome-wide MITF DNA-binding affinity, it preferentially directs DNA binding away from differentiation-associated CATGTG motifs toward CACGTG elements. The results reveal an acetylation-mediated switch that suppresses differentiation and provides a mechanistic explanation of why a human K206Q MITF mutation is associated with Waardenburg syndrome. 


\section{INTRODUCTION}

The gene expression programs that underpin development and homeostasis are mediated by the activity of sequence specific transcription factors that up- or down-regulate their target genes in response to intra- and extracellular cues. De-regulation of signals that are interpreted by transcription factors can lead to altered gene expression programs associated with diseases such as cancer. The ability of any given transcription factor to find its target elements in the genomic context is affected by several factors including the affinity of the transcription factor for DNA, its abundance, and whether its cognate recognition element is accessible or occluded by nucleosomes ${ }^{2-4}$. Importantly, the same transcription factor may regulate different sets of target genes with fundamentally different biological functions, implying that their targeting may be regulated. How transcription factors distinguish between different repertoires of binding sites in response to cell extrinsic or intrinsic cues is poorly understood.

The microphthalmia-associated transcription factor MITF ${ }^{5}$ plays a critical role in development of the melanocyte lineage where it controls survival of melanoblasts ${ }^{6}$. Consequently reduced expression or mutation can lead to Waardenburg syndrome, characterized by pigmentation defects ${ }^{7}$. MITF has a critical coordinating role in regulating many cellular functions, and in melanoma, a highly aggressive skin cancer originating in melanocytes, MITF has been defined as a lineage survival oncogene ${ }^{8}$. In addition to up-regulating a repertoire of melanocyte differentiation-associated genes, MITF suppresses invasion and senescence and drives proliferation, lysosome biogenesis and autophagy, and reprograms metabolism ${ }^{9-23}$. To account for its apparently contradictory role in promoting both differentiation and proliferation, a rheostat model was proposed in which low levels of MITF activity are associated with invasion, intermediate activity with proliferation, and high activity drives differentiation ${ }^{11}$. Yet while the rheostat model is a useful approximation to explain MITF's role in cell biology ${ }^{24}$, it does not explain how differentiation is prevented in proliferating cells. One clue may be provided by the target sequence specificity of MITF. As a basic-helix-loop-helix-leucine zipper (bHLH-LZ) transcription factor MITF recognizes 6 bp E-box motifs, with 5' flanking T residues that facilitate recognition by MITF and prevent binding by MYC ${ }^{25-29}$. MITF recognizes both core CACGTG and CATGTG E-box elements, with the latter being found in differentiation-associated genes such as TYR and $A B C B 5^{30-32}$. Whether or how MITF might differentiate between these two classes of target site is unknown, but is important since preventing binding to CATGTG elements could in principle lead to de-differentiation. One plausible model is that stable recognition of differentiation genes that contain CATGTG M-box motifs, but not CACGTG E-boxes, is prevented by MITF post-translational modification of residues implicated in direct DNA binding, though to date no such MITF modification has been identified.

In melanoma, the major driver mutations in BRAF and NRAS lead to activation of the MAPK pathway, and ERK-mediated phosphorylation of MITF has been variously reported to control its nuclear export ${ }^{33}$, stability ${ }^{34}$ and recruitment of the acetyl transferases $\mathrm{CBP} / \mathrm{p} 300{ }^{35}$. Moreover, increased pro- 
proliferative MAPK signaling increases acetylation by promoting p300/CBP activity ${ }^{36}$. Although it remains unclear if or how deregulated MAPK signaling might enable MITF to differentiate between its targets implicated in proliferation versus those driving differentiation, understanding how MITF activity is regulated is especially important in melanoma as varying MITF activity has been associated with drug and immunotherapy resistance ${ }^{15-18,37-41}$.

Here we reveal that compared to many transcription factors MITF exhibits a long $(>100 \mathrm{~s})$ residence time which is decreased by acetylation at MITF K206. Notably acetyl K206 preferentially decreases MITF DNA-binding affinity for differentiation-associated CATGTG elements and shifts the equilibrium of MITF binding towards CACGTG motifs. The results highlight a mechanism by which activation of MAPK signaling would prevent melanocyte differentiation, and may also explain why a human K206Q mutation leads to Waardenburg syndrome.

\section{RESULTS}

Like all bHLH-LZ factors, DNA-binding by MITF is mediated by the basic region that makes basespecific contacts, that determine target sequence specificity, as well as non-specific contacts mediated by basic amino acid interaction with the phosphate backbone ${ }^{42}$. In considering the possible mechanisms by which bHLH-LZ factor target gene selectivity could be regulated, we hypothesized that a posttranslational modification affecting amino acids directly contacting DNA could be important in either changing MITF target specificity directly, or by affecting MITF's DNA binding affinity and indirectly modulating its capacity to bind selectively different elements.

Consistent with previous observations that MITF can be acetylated by the p300 and CBP acetyl transferases ${ }^{30}$, well-established MAPK-activated cofactors for MITF ${ }^{35,43}$, western blotting using a pananti-acetyl lysine antibody of immunoprecipitated GFP-tagged MITF co-expressed with either the CBP, p300 or GCN5 acetyl transferases revealed acetylation of MITF, but not GFP when MITF was coexpressed with CBP or p300. No significant acetylation was detected when GCN5 was co-expressed (Fig. 1a). Mass spectrometry analysis of immunoprecipitated MITF revealed acetylation of four residues, K33, K91, K206 and K243 (Fig. 1b, Supplementary Fig. 1), but no other modification (e.g. methylation) on these sites was detected in this or other experiments.

Of the acetylated residues identified only K206 lies within the basic region that determines MITF sequence-specific DNA-binding activity. This residue is highly conserved between species and in all members of the MITF subfamily of bHLH-LZ transcription factors including TFEB and TFE3 (Fig. 1c), but not in other bHLH-LZ proteins such as USF1, MYC and MAX, suggesting it may play a distinct regulatory role in the MITF-subfamily. Moreover, a DNA-MITF DNA-binding domain (DBD) cocrystal structure indicates K206 contacts the phosphate backbone within the basic region ${ }^{42}$ (Fig. 1d). Significantly, a heterozygous lysine to glutamine substitution (K206Q), that is widely accepted as an acetylation mimetic, has been identified in a family with Waardenburg syndrome Type $2^{44}$, characterized by pigmentation abnormalities and hearing loss owing to defective melanocytes in the 
inner ear. Collectively these observations suggest that acetylation of K206 could potentially play a key role in modulating MITF function.

To validate the mass spectrometry results we generated an anti-acetyl K206 antibody and tested its activity against a range of MITF peptides. The results revealed that the antibody was highly selective, strongly recognizing peptides containing acetyl-K206, and only weakly binding a peptide containing acetyl K33 (Fig. 1e). No activity was detected against non-acetyl K206, nor against a range of other acetyl or non-acetyl control peptides derived from MITF or histone H3. To confirm acetylation of MITF at K206, we immunoprecipitated HA-MITF co-expressed with CBP or p300, with or without the selective $\mathrm{p} 300 / \mathrm{CBP}$ inhibitor A485. The results revealed that in the input control, $\mathrm{p} 300$ or CBP increased global acetylation detected by a pan-acetyl-lysine antibody that was diminished by A485 (Fig. 1f, upper panels). After immunoprecipitation of MITF using an anti-HA antibody, probing with the anti-acetyl MITF K206 antibody revealed MITF acetylation at this site was decreased in the presence of A485 (Fig. 1f, lower panel). Immunoprecipitation of MITF from an IGR37 human melanoma cell line engineered to inducibly express p300 and HA-MITF revealed an increase in acetyl K206 MITF after induction using doxycycline (Fig. 1g), confirming a role for p300 in MITF acetylation. We were also able to detect acetylation of endogenous MITF after immunoprecipitation using anti-MITF antibody and an anti-HA antibody as an isotype control (Fig. 1h). While anti-HA failed to immunoprecipitate endogenous MITF as expected, the anti-MITF antibody pulled down MITF (bottom left panel) that when probed with the anti-acetyl K206 antibody (right panel) revealed that MITF is acetylated at this residue. Since p300 acetyl transferase activity is activated by MAPK signaling ${ }^{30,36}$ downstream from BRAF or NRAS, we also repeated the immunoprecipitation experiment using HA-MITF from cells treated or not with the MEK inhibitor U0126. The results indicated that MITF acetylation at K206 was reduced when the MAPK pathway was inhibited (Fig. 1i). Finally, we repeated the experiment using a 501mel cell line stably expressing HIS-tagged MITF at endogenous levels ${ }^{30}$. This enables the direct purification of MITF on nickel beads after solubilization of cellular proteins using 6 $\mathrm{M}$ guanidinium $\mathrm{HCl}$. In this experiment, $200 \mathrm{nM}$ TPA, previously shown to promote melanogenesis ${ }^{45}$, was used to increase MITF expression. The result (Fig. 1j) confirmed that MITF K206 is acetylated in the absence of ectopic p300 or CBP.

\section{Single molecule tracking reveals K206 regulates MITF chromatin association in vivo}

MAPK-dependent acetylation of MITF at K206 is likely to have functional consequences, in part because of the pigmentation defects in human K206Q heterozygotes. Because K206 makes a phosphate backbone contact in the DNA co-crystal structure, we asked whether a K206R non-acetylatable mutation or the K206Q mutation that mimics constitutive acetylation might affect the ability of MITF to bind DNA. To do this we initially used a single molecule tracking (SMT) assay ${ }^{46}$ to examine MITF WT and K206 mutant dynamics and their chromatin association in live cells. By fusing MITF to the HALO tag labelled with a bright, photostable fluorescent ligand, JF594 ${ }^{47}$ at sub-saturating concentrations we can estimate the duration of binding events, the fraction of immobilized MITF bound to chromatin, and the 
diffusion properties of the protein ${ }^{48-50}$. We therefore established a 501 mel human melanoma cell line stably expressing doxycycline-inducible HALO-tagged MITF WT and K206 mutants (Fig. 2a). We also included as an additional control a non-DNA-binding mutant lacking the MITF basic region that makes direct DNA contacts. In all constructs we also included the SV40 T-antigen nuclear localization signal ${ }^{51}$ since perturbation of the MITF basic region can affect nuclear import ${ }^{52}$. We then used $20 \mathrm{ng}$ doxycycline to induce expression of the HALO tagged MITF WT and K206 mutants and imaged single MITF molecules upon two different regimes. First, in order to evaluate how K206 acetylation affects the mobility of the MITF we acquired movies at $100 \mathrm{fps}$, using a $5 \mathrm{~ms}$ laser exposure. Single molecule tracks were analyzed in terms of the distributions of displacements (Fig. 2b), which were fit with a three component diffusion model as previously described ${ }^{53-55}$, where the slowest component describes immobilized molecules (on chromatin or other nuclear structures), and the two others represent diffusing MITF, possibly slowed down by transient interactions (faster than the acquisition framerate). The results for WT MITF indicated that $35 \pm 2 \%$ of molecules were immobilized, $51 \pm 1 \%$ were found in a slow diffusion state with $\mathrm{D}_{\text {slow }} \sim 0.1 \mu \mathrm{m}^{2} / \mathrm{s}$, and the remaining molecules were in a fast diffusion state with $\mathrm{D}_{\text {fast }} \sim 0.75 \mu \mathrm{m}^{2} / \mathrm{s}$ (Fig. 2c, d). Compared to WT MITF, the K206R mutant displayed a minor decrease in immobile fraction to $30 \%$ and a corresponding increase in the fast diffusing fraction to $19 \%$, but no significant change in the slow diffusing fraction (Fig. 2c). The MITF K206Q mutant exhibited a similar bound fraction to the K206R mutant, but a more pronounced increase in its fast diffusing fraction up to $30 \%$ at the expense of the slow-diffusing fraction (Fig. 2c). These data indicate that the K206Q mutant is generally more mobile than both WT MITF and MITF-K206R.

To better characterize MITF binding kinetics, we next collected single molecule movies at $2 \mathrm{fps}$, using a laser exposure of $200 \mathrm{~ms}$ to blur out the diffusing molecules ${ }^{56}$ and to quantify the dwell times of immobilized molecules. Since the measurements taken over a long period can be affected by photobleaching, we used a histone H2B-HaloTag as an additional control since a large quota of H2B is stably incorporated into chromatin, and then corrected the MITF residence times using the slowest decay rate observed for H2B-HaloTag, as recently described ${ }^{57}$. The results shown in Fig. 2e, with quantification of binding events lasting more than 100s shown in Fig. 2f, enable us to generate several conclusions. First, based on the results corrected for photobleaching, around 32\% of bound WT MITF exhibits a long residency time in excess of 100 seconds. Second, the quota of long-lived binding events decreases for the K206Q (to 22\%), whereas the K206R mutant binds even tighter with nearly $46 \%$ of molecules stably bound, consistent with a proportion of MITF being acetylated in vivo. Third, the $\Delta$ basic DNA-binding MITF mutant displays more transient immobilization events than WT MITF and MITFK206 mutants, although a proportion (about 9\%) of the protein still exhibits a residence time of greater than 100 seconds. This indicates that the majority of the stably bound MITF is likely interacting with DNA via the basic region, but a contribution of long-lived residence time is made by residues outside of the basic region that presumably participate in protein-protein interactions. Of note, another bHLH- 
LZ TF, USF1, as well as an unrelated TF, p53, display a much faster dissociation from chromatin than WT MITF, indicating that tight binding to DNA in vivo might be a peculiar property of MITF. This is consistent with previous observations ${ }^{30}$ indicating that unlike USF1, MITF is poorly extractable from cell nuclei even under very harsh conditions. Together the data indicates that the K206Q mutant binds chromatin less tightly than WT MITF. The K206R mutant instead appears to only bind a subset of the sites bound by MITF-WT (as the bound fraction of WT MITF is higher than that measured for MITFK206R), but the binding at these sites is more stable (as a larger quota of MITF-K206R exhibit binding lasting longer than 100s). Since the proportion of WT MITF with long residence times is between that observed for K206R and K206Q, the results are also consistent with a proportion of WT MITF being acetylated in cells.

\section{Acetylation of MITF K206 differentiates between M-box and Clear-box motifs}

To determine the effect of acetylation of K206 on MITF DNA-binding directly we bacterially expressed the MITF DNA-binding and dimerization domain (DBD) and used the purified protein in in vitro DNA-binding assays. A modified bacterial expression system, in which introduction of an amber stop codon into the K206 position enabled acetylation of K206 to be genetically encoded, allowed us to express and purify the MITF DNA-binding domain (DBD) specifically acetylated to high efficiency at K206 (Supplementary Fig. 2). We also expressed and purified the wild-type (WT) MITF DBD, a nonacetylatable K206R mutant, and the Waardenburg-associated K206Q mutant that was expected to mimic acetylation by breaking the phosphate backbone contact. These were then used in fluorescence anisotropy assays together with three fluorescently labeled oligonucleotides. These contained the full palindromic 8 bp TCACGTGA binding motif termed the CLEAR-box that is associated with genes other than those implicated in pigmentation; a related TCATGTGA element associated with the melanocytespecific TYROSINASE (TYR) enhancer that retained the flanking 5'T and 3'A bases important for MITF binding ${ }^{58}$; and the TCATGTGT M-box sequence ${ }^{59}$ found in all differentiation-associated promoters. Increasing the amounts of the purified WT, K206R and K206Q mutants and Ac-K206 proteins allowed us to determine the relative affinities for each type of element. Examples of the binding curves are shown in Fig. 3a and a summary of the relative affinities derived is presented in Fig. 3b. The bacterially expressed and therefore non-acetylated WT MITF exhibited only a moderate reduction in affinity between the CLEAR-box and TYR enhancer, but has over a 10-fold reduced affinity for the promoter M-box motif. Similar results were obtained using the non-acetylatable K206R mutant. By contrast AcK206 MITF clearly distinguished between the two sequence elements: binding to the TCACGTGA CLEAR-box was reduced around 3-fold compared to the non-acetylated WT protein, but was diminished a further 5-fold using the TYR TCATGTGA motif while binding to the M-box was 20-fold reduced compared to the CLEAR box. The effect of acetylation on MITF DNA binding was largely reproduced using the K206Q mutant. These results revealed that while acetylation of MITF K206Q moderately reduces binding to the CLEAR box, binding to the M-box present in differentiation-associated promoters 
was much more severely affected. Thus, modification of K206 can potentially enable MITF to discriminate between these two classes of regulatory element. The reduced DNA binding affinity of the K206Q mutant, reflected in its reduced residence time determined using SMT analysis, may therefore account for the Waardenburg disease associated with this variant.

\section{Modification of MITF K206 affects melanocyte development}

Since Ac-K206 MITF exhibits reduced DNA-binding affinity we anticipated that the Waardenburgassociated K206Q mutation would exhibit defects in melanocyte development. To test this, we used a zebrafish assay in which MITF WT or mutants are used to complement the absence of MITF in an mitfanull nacre zebrafish that lacks all neural crest-derived melanocytes ${ }^{60}$. To this end, WT or K206 mutant (K201 in fish) zebrafish MITF were transiently expressed from the fish mitfa promoter (Fig. 4a) and the effects on melanocyte number in the larval fish assessed 5 days post-fertilization. The results (Fig. 4b, c) revealed that whereas WT and K201R mutant MITF complemented the absence of endogenous MITF and generated a normal pattern of melanocytes, the K201Q mutant failed efficiently to complement the absence of MITF, consistent with it possessing a defective capacity to regulate gene expression.

\section{K206 modification regulates genome-wide distribution of MITF}

The results so far suggest that acetylation of MITF would lead to reduced DNA binding overall, but that binding to differentiation-associated genes would be significantly more affected. Since binding of the K206Q mutant accurately mimicked binding of the acetylated K206 protein in vitro, we next established human 501mel melanoma cells in which WT MITF, as well as the K206Q and K206R mutants were stably expressed from a doxycycline-inducible promoter. By using an inducible promoter, we were able to carefully titrate the levels of MITF WT and mutants expressed (Fig. 5a). Since the ectopic HA-epitope tagged MITF migrates slower when analyzed by SDS PAGE, we determined that $20 \mathrm{ng}$ doxycycline induced a similar level of ectopic WT and mutant MITF to the endogenous protein present in uninduced $501 \mathrm{mel}$ cells (Supplementary Fig.3a). Using these inducible MITF cell lines we performed duplicate ChIP-seq experiments at 0, 20 and $100 \mathrm{ng}$ doxycycline for the WT and each mutant, with the replicate results obtained showing high concordance (Supplementary Fig. 3b).

As expected, the read density of the WT MITF ChIP showed that increasing the levels of doxycycline led to increased MITF DNA binding reflected in an increasing peak score (Fig. 5b, left panel), an increase in total numbers of peaks called and corresponding increase in concordance between the replicates (Supplementary Fig. 3c). This was reflected in binding at individual genes known to be MITF targets (Fig. 5c, left panels; Supplementary Fig. 3d, left panels). For example, at 0 ng doxycycline, when the HA-tagged MITF is undetectable by western blotting (Fig. 5a), a highly specific ChIP signal is observed at the BLOCIS and MLANA genes that contain TCACGTGA or BCACGTGA MITF binding sites respectively, but a peak was not readily discernable at $D C T$ with a TCATGTGC site. As the level of MITF is increased using $20 \mathrm{ng}$ or $100 \mathrm{ng}$ doxycycline the peak score increases at BLOC1S and at 
$M L A N A$, and at $D C T$ specific binding now becomes apparent. As shown for other transcription factors ${ }^{3}$, most likely nucleosome positioning or cooperating co-factors make significant gene-specific contribution to binding affinity at any locus. Nevertheless, the results indicate that increasing MITF levels, for example as observed during melanocyte differentiation in response to MC1R signaling, allows binding to differentiation-associated genes such as $D C T$ that is not detected at lower levels of MITF.

We next compared the binding of WT MITF to that of the non-acetylatable K206R mutant and the Waardenburg-associated K206Q acetylation mimetic. Like the WT, binding by the K206R mutant was detected at the lowest concentration of MITF to genes such as BLOCIS or MLANA, though not at DCT (Fig. 5c, middle panels). At $20 \mathrm{ng}$ doxycycline, when ectopic MITF was expressed to levels similar to the endogenous protein, at many genes K206R bound better than WT (Fig. 5c,d; Supplementary Fig. 3d), consistent with its increased residence time determined using SMT analysis (Fig. 2e). By contrast, the K206Q mutant exhibited a decrease in global DNA binding, consistent with its reduced residence time in cells (Fig. 2e, f) and lower DNA binding affinity in vitro (Fig. 3). While binding was detected by K206Q at BLOC1S and MLANA, with a peak height around 30\% of WT at $20 \mathrm{ng}$ doxycycline, binding to the differentiation-associated genes DCT (Fig. 5c, lower panel) or PMEL (Supplementary Fig. 3c, lower right panel) was barely detected above background at any concentration of doxycycline. Given that at $20 \mathrm{ng}$ doxycycline binding by WT MITF was intermediate between that of the non-acetylatable K243R mutant and the acetylation mimetic K243Q mutant, our results are consistent with a significant proportion of MITF being acetylated in 501mel cells, as observed using the anti-acetyl K206 antibody (Fig. 1h).

The DNA-binding affinity measurements (Fig. 3) indicated that acetyl K206 MITF or the K206Q mutant can distinguish between a CACGTG motif and the lower affinity CATGTG E-box. To determine whether the K206Q and K206R mutants differentially recognized these motifs in cells, we identified the sequences beneath the peaks after induction using 0,20 or $100 \mathrm{ng}$ doxycycline. The results for the WT protein indicated that as MITF levels were increased, there was an increase in the proportion of lower affinity binding sites recognized, with a shift away from the canonical TCACGTGA motifs towards TCACGTGB or TCATGTGA elements (Fig. 5e). As expected the K206R mutant behaved similarly to the WT protein, although at $0 \mathrm{ng}$ doxycycline there was a greater proportion of TCACGTGA motifs bound. By contrast, although a largely similar shift was observed on increasing the levels of the K206Q mutant, the vast majority of sites recognized were TCACGTGA. These observations were confirmed when analyzing the global consensus beneath the WT and mutant MITF peaks at 20 ng doxycycline that revealed that the consensus for the WT and K206R mutants reflected a mix of CACGTG and CATGTG core E-box motifs, while that of the K206Q mutant was restricted to the high affinity CACGTG element (Fig. 5f). A similar result was obtained by analysis of peak score versus motif incidence that revealed that the WT and K206R mutants behaved similarly, though with the K206R mutant exhibiting somewhat 
better binding than WT MITF (Fig. 5g). Again, the K206Q mutant bound fewer sites and those that were bound were primarily CACGTG motifs.

\section{DISCUSSION}

Transcription factors play a critical role in establishing and maintaining specific cellular phenotypes. To do so they must identify specific sets of binding sites in genes that determine cell identity. In melanoma, MITF has been implicated in regulating many biological processes including reprogramming metabolism, promoting survival, proliferation, differentiation and autophagy, and suppressing invasion and senescence ${ }^{5}$. The current rheostat model of MITF function suggests that different levels of MITF expression or activity enables cells to adopt distinct phenotypes ${ }^{11}$. This model is supported by the observation that a reduction in MITF function using a temperature sensitive mutant in zebrafish causes differentiated melanocytes to re-enter the cell cycle ${ }^{61}$ and that MITF-independent states characterise persister states in residual disease that are similar to those generated in response to BRAFi ${ }^{1}$. Our results suggest that the CATGTG M-box motifs associated with the promoters of differentiation genes are lower affinity binding sites, whereas CACGTG E-boxes are higher affinity and can therefore be recognized at lower protein levels. However, in addition to MITF levels, the ability of MITF to control its target genes will also be regulated by post-translational modifications.

Significantly we find that the ability of MITF to bind DNA is inhibited by CBP/p300-mediated acetylation of K206. This residue lies within the basic region that mediates both base-specific contacts to provide sequence specific binding, as well as phosphate backbone interactions that increase DNA binding affinity without directly affecting base recognition. However, while acetylation of K206, that makes a phosphate backbone contact, moderately reduces the affinity to a CACGTG E-box or TCACGTGA enhancer element, it drastically reduces binding to CATGTG M-box motifs. The reduction in Ac-K206 DNA binding in vitro was confirmed in cells using a K206Q mutant and SMT analysis. Notably, the in vitro binding results were reflected in vivo using ChIP-seq where recognition of CATGTG motifs by the K206Q mutant was largely blocked while binding to genes containing CACGTG elements was retained, though with a reduced peak height. The ability of K206 acetylation to differentiate between CATGTG and CACGTG elements suggests that regulation of MITF acetylation may be instrumental in determining MITF's repertoire of target genes. Consistent with activation of BRAF or NRAS upstream from ERK promoting melanocyte proliferation and de-differentiation, MITF interaction with $\mathrm{CBP} / \mathrm{p} 300$ is promoted by ERK-mediated phosphorylation of $\mathrm{MITF}^{35}$ and $\mathrm{CBP} / \mathrm{p} 300$ acetyl transferase activity is stimulated by MAPK signaling ${ }^{30,36}$. However, the activity of CBP/p300 is also inhibited by deacetylation mediated by SIRT1 ${ }^{62}$, an MITF-activated target gene ${ }^{63}$, suggesting that regulation of MITF acetylation by $\mathrm{CBP} / \mathrm{p} 300$ may be subject to an autoregulatory feedback loop.

Our results also suggest that MITF may represent a distinct class of transcription factor that is tightly bound within the nucleus through both DNA and non-DNA interactions with over $30 \%$ of molecules exhibiting a residence time of greater than 100 seconds, a proportion significantly increased when the 
non-acetylatable K206R mutant is used. This is remarkably long for a transcription factor where SMT has determined typical residence times of few seconds ${ }^{64}$. The known exceptions are the serum response factor SRF after serum stimulation in fibroblasts $\left(\tau_{\text {res }} 1-4 \mathrm{~min}\right){ }^{65}$, polycomb repressive complex 1 (PRC1) $\left(\tau_{\text {res }}>100 \mathrm{sec}\right){ }^{66}$ and CTCF ( $\tau_{\text {res }}$ around $\left.60-120 \mathrm{sec}\right){ }^{67,68}$. The long residency time of MITF is not related to the structure of the MITF DNA binding and dimerization domain nor the E-box motifs recognized, since USF1, which also contains a bHLH-LZ motif and can bind the same sites as MITF ${ }^{69}$, is readily extracted from nuclei and binds with a residence time distribution similar to that of the K206Q MITF mutant. It seems likely therefore, that a significant contribution to MITF's residency time is made by protein-protein interactions. Given the role of CTCF in determining and stabilizing chromatin topology though binding to boundary elements ${ }^{68,70}$, and the role of PRC1 in 3D chromatin organization and control of cell identity ${ }^{71-74}$, it is plausible that MITF's tight association with nuclear structures ${ }^{30}$ and long residency times reflects a similar role in establishing chromatin conformations that determine the melanocyte lineage. This would be consistent with observations made on the residence time of the neuronal lineage-determining factor Ascl1 using in vivo competition assays ${ }^{75}$. These authors suggested that the long residence time of Ascl1 in non-dividing cells, that was in the order of hours, might be important for securing the differentiated state of cells, while in proliferating cells residence times would be reduced to generate flexibility in the transcriptional response. In this respect we note that acetylation by $\mathrm{p} 300 / \mathrm{CBP}$ is controlled by pro-proliferative MAPK signaling ${ }^{30,36}$. Consequently, in non-dividing differentiated cells where MAPK signaling will be reduced, it seems likely that MITF's long residence time will be further increased, as is observed using the K206R mutant. Moreover, it is possible that if endogenous MITF has an extremely long residence time, its ability to be displaced by expression of ectopic MITF would be reduced. If so, this might explain the observations ${ }^{76,77}$ that while depletion of MITF leads to a reduction in expression of its target genes, ectopic MITF fails to regulate the same genes.

Finally, in addition to deciphering how K206 acetylation affects MITF target specificity, our results may also be relevant for understanding the genetics of Waardenburg's disease. In this respect, it is clear that the Waardenburg's-associated K206Q mutation ${ }^{44}$, by mimicking K206 acetylation leads to reduced MITF DNA residence time and consequently to a diminished capacity to promote the biological functions associated with MITF leading to the developmental defects associated with the disease.

In summary, our results highlight a mechanism by which post-translational modification of MITF regulates its ability to bind its targets and thereby control its ability to coordinate the gene expression programs that underpin melanocyte and melanoma proliferation and differentiation.

\section{METHODS}

\section{Plasmids}


pCS2-6xMyc-MITF used for mass spectrometry has been described previously ${ }^{78}$. pCMV5-3xHA-Mitf was PCR cloned from Mitf cDNA using EcoRI (NEB; Cat\#R3101L). pEGFP-Mitf was generated via subcloning from pCMV5-3xHA-Mitf using EcoRI into pEGFP-N1 (Clontech). pETM-11MITF $\Delta$ N180 $\Delta$ C296 has been described ${ }^{42}$, as has the doxycycline inducible PiggyBac 3xHA-Mitf expression vector used for ChIP-seq ${ }^{18}$, and the 3xHA-MITF-3xFLAG-6xHIS used for acetylation analysis and the Halo-tagged WT MITF ${ }^{30}$. The K206 mutants were derived by site-directed mutagenesis (Agilent \#210519). pcDNA3-3×HA-p300 was a gift from Bernhard Luescher. All plasmids have been sequenced and verified prior to use.

\section{Cell lines}

$501 \mathrm{mel}$ and its derivative cell lines were maintained in RPMI-1640 (Gibco\#61870-010), 10\% fetal bovine serum (FBS, Biosera) at $37^{\circ} \mathrm{C}$ in humidified incubator with $10 \% \mathrm{CO}$. Reverse-transfection was perform using 3:1 ratio of FUGENE VI (Promega \# E2692):DNA suspended in Opti-MEM (GIBCO \#31985-062) according to the supplier's protocol. Stable inducible cell lines were generated from transiently-transfected cells using Geneticin (1 mg/ml) (GIBCO \#10131-027), Puromycin (5 $\mu \mathrm{g} / \mathrm{ml})$ (GIBCO \#A11138-02).

All cell lines were tested monthly for mycoplasma and authenticated using short tandem repeat analysis.

\section{Antibodies}

Primary antibodies used were $\alpha$-Acetylated-Lysine (RRID: AB_331805), MITF (RRID: AB_570596), HA (RRID: AB_514506), GAPDH (RRID: AB_627679) and GFP (RRID:AB_303395). MITF-K206Ac antibody was raised in-house. Secondary HRP antibodies were procured from Biorad (\#170-6516 \& \#170-6515). Secondary Alexa dye-labelled antibodies were procured from Invitrogen (\#A21202 \& A10040).

Zebrafish Plasmid DNA $(62.5 \mathrm{ng} / \mu \mathrm{l})$ comprising the zebrafish promoter driving the fish MITF cDNA was mixed with Tol2 mRNA $(75 \mathrm{ng} / \mu \mathrm{l}) .2 \mathrm{nl}$ of the mixture was injected into 1-cell stage mitf-null nacre embryos. Injected embryos were grown at $28^{\circ} \mathrm{C}$ for 5 days. On day 5 , embryos were briefly exposed to white light to contract melanocytes and were then imaged before being fixed in $4 \%$ PFA. The total number of surface melanocytes in the head, trunk and yolksac was counted. All zebrafish experiments are performed in accordance with the Animals (Scientific Procedures) Act 1986, and approved by the University of Edinburgh Animal Welfare and Ethical Review Body. Zebrafish AB/TPL lines were bred, raised and maintained as described ${ }^{79}$. 
Western blotting Whole cell extract (WCE) were prepared from, trypsinised, washed and centrifuged cell pellets dissolved in $4 \times$ Laemmli sample buffer or $4 \times$ NuPAGE® LDS Sample Buffer (Novex ${ }^{\circledR}$ $\#$ B0008) $+20 \% \beta$-mercaptoethanol, added fresh before use. Extracts were boiled at $95^{\circ} \mathrm{C}$ for 5 min. SDS-PAGE were carried out on Bis-Tris gels, except when MITF-hyperphosphorylation is to be separated, the samples were run on $12.5 \%$ Tris-Glycine made with 200:1 acryamide:bisacrylamide (custom order, Severn Biotech). Gels were transferred onto Immun-Blot Low Fluorescence PVDF Membrane (Biorad) at $100 \mathrm{~V}$ for $60 \mathrm{~min}$ for Western-blotting. Blotting involved blocking for $1 \mathrm{~h}$, room temperature in 5\% non-fat milk in TBS containing 0.25\% TWEEN-20 (TBST). Antibodies incubation were done in $5 \% \mathrm{BSA}-\mathrm{TBST}$ overnight, $4^{\circ} \mathrm{C}$ for primary antibodies and $1 \mathrm{~h} \mathrm{RT}$ for secondary antibodies. Visualization was carried out on X-ray film using ECL (GE) or ChemiDoc (Biorad).

Immunoprecipitation All steps involved in affinity purifications were carried out at $4{ }^{\circ} \mathrm{C}$ using LoBind Tubes (Eppendorf\#Z666505-100EA). Transiently transfected cells were trypsinised, washed in PBS and pelleted by centrifugation at $1200 \times \mathrm{g}$ for $4 \mathrm{~min}$. Cell pellets were lysed in RIPA supplemented with $4 \times$ protease inhibitor cocktail (Roche\# 11836145001). The cell suspensions were briefly sonicated for $30 \mathrm{~s}$ ( $7.5 \mathrm{~s}$ on/off cycle) using Bioruptor ${ }^{\circledR}$ Plus (Diagenode) then cleared by centrifugation at $14000 \times \mathrm{g}$ for $10 \mathrm{~min}$. The supernatants were transferred to fresh tube, keeping $10 \%$ as input and the remainder rotated overnight with $20 \mu \mathrm{g}$ antibody. An appropriate amount (10\% on top of the theoretical binding capacity) of Dynabeads Protein G (Invitrogen\#10004D) were washed in lysis buffer and blocked overnight with $0.5 \mathrm{mg} / \mathrm{ml} \mathrm{BSA}$ (Sigma \#B8894) before adding to the samples and rotate for $1 \mathrm{~h}$. The beads were washed 6 -times with lysis buffer, transferring to a new tube after the first wash and finally boiled in $4 \times$ LDS.

GFP-tag affinity-purification GFP pull-down samples were purified using from Phoenix-ampho cell line transiently transfected $(16 \mathrm{~h}$ ) with $6 \mu \mathrm{g}$ of the indicated plasmids in a $6 \mathrm{~cm}$ petri dish using FuGENE VI as described above. Cells for each experiment were lysed in $500 \mu 1$ RIPA supplemented with cOmplete ${ }^{\text {TM }}$ ULTRA protease inhibitor cocktail (Sigma Cat\#5892988001) and $5 \mu$ M M344 (Stratech Scientific Cat\#S2779-SEL) on ice for $30 \mathrm{~min}$ and subjected to mechanical disruption using 25 guage needle till no visible clump can be seen. $100 \mu 1$ of washed, pre-equilibrated GFP-trap (chromotek Cat\#gtma-100) in lysis buffer was added to each precleared (14,000 $\mathrm{x} g$ for $10 \mathrm{~min})$ sample, incubated overnight, $4^{\circ} \mathrm{C}$, washed thrice in RIPA without NP-40 and eluted in LDS, $95^{\circ} \mathrm{C}$ for $10 \mathrm{~min}$ before SDSPAGE.

His-tag affinity-purification Cells were treated with $5 \mu \mathrm{M}$ M344 (Tocris\#2771) and $200 \mathrm{nM}$ TPA (Sigma\#4174S) at the indicated time before lysis in $1 \mathrm{ml}$ His-purification lysis buffer (6 M guanidinium $\mathrm{HCl}, 0.1 \mathrm{M} \mathrm{Na}_{2} \mathrm{HPO}_{4} / \mathrm{NaH}_{2} \mathrm{PO}_{4}, 10 \mathrm{mM}$ Tris- $\mathrm{HCl}$ (pH8), $0.005 \mathrm{M}$ imidazole, 0.01M $\beta$-ME, 1x EDTAfree protease inhibitor cocktail (Roche\# 5892791001) for 5 min room-temperature before preclearing by centrifugation at $14000 \times \mathrm{g}$ for $10 \mathrm{~min}, 4^{\circ} \mathrm{C}$. Supernatant is then transferred to $15 \mathrm{ml} \mathrm{Falcon}$ tube 
(Sigma\#CLS430052), topped up with $4 \mathrm{ml}$ His-purification lysis buffer and rotate overnight, $4^{\circ} \mathrm{C}$ in $\mathrm{Ni}$ NTA resin (Merck\# 70691-3). Ni-NTA resin was equilibrate in 2 times bead volume in His-purification lysis buffer for $5 \mathrm{~min}$, room-temperature before adding to the lysate. The beads were washed once in His-purification lysis buffer and three times in His-purification wash buffer $(8 \mathrm{M}$ Urea, $0.1 \mathrm{M}$ $\mathrm{Na}_{2} \mathrm{HPO}_{4} / \mathrm{NaH}_{2} \mathrm{PO}_{4}, 10 \mathrm{mM}$ Tris- $\mathrm{HCl}(\mathrm{pH} 8), 0.005 \mathrm{M}$ imidazole, $0.01 \mathrm{M} \beta-\mathrm{ME}$ ) rotating $5 \mathrm{~min}$ between each wash. The washed-resins were directly eluted in LDS buffer.

Peptide array Peptides were printed on cellulose membranes using SPOT technology ${ }^{80}$ with 9 amino acids to the left and the right of the indicated acetylated or non-acetylated residue. Membranes were activated in EtOH and rinse thoroughly with TBST prior to blotting. The array blotting was carried out as with Western-blotting, except the blocking was carried out for $4 \mathrm{~h}$.

Mass spectrometry and sample preparation HEK 293 cells were transfected with a mammalian Mycepitope-tagged murine MITF expression vector together with expression vectors for either CBP or p300. $48 \mathrm{~h}$ post-transfection cells were harvested, and whole cell lysate used for immunoprecipitation using anti-Myc antibody. Immunoprecipitates were resolved using SDS PAGE and gel bands corresponding to MITF were excised, destained then reduced and alkylated and digested with chymotrypsin overnight. Digests were analysed using a LTQ XL Orbitrap (Thermo, Hemel Hempstead), coupled to a Dionex Ultimate 3000 nano HPLC system (Camberley, Surrey). Data were analysed using Mascot (Matrixscience, London). Precursor mass tolerance was set to $10 \mathrm{ppm}$, fragment mass tolerance was 0.5 $\mathrm{Da}$, fixed modification was carbamidomethylation of cysteine and variable modification was oxidised methionine and acetylated lysine. Data were searched against an IPI mouse database.

Recombinant protein purification MITF DNA-binding domains (residues 180-296) that were either WT, K206Q or K206R were expressed in Escherichia coli BL21(DE3) cells (Agilent Technologies). Cultures were grown in Luria-Bertani broth to an $\mathrm{OD}_{600}$ of 0.7-0.8. Recombinant protein overexpression was induced by addition of isopropyl $\beta$-D-1-thiogalactopyranoside (IPTG; $0.5 \mathrm{mM}$ final concentration) and the cultures were incubated for a further $6 \mathrm{~h}$. Cells were harvested by centrifugation, washed in PBS and frozen on dry ice. After thawing, cells were suspended in lysis buffer $\left(50 \mathrm{mM} \mathrm{NaH}_{2} \mathrm{PO}_{4}, 300 \mathrm{mM}\right.$ $\mathrm{NaCl}, 10 \mathrm{mM}$ imidazole, 10\% v/v glycerol, $\mathrm{pH} 7.4$ [NaOH], $20 \mathrm{mg} / \mathrm{ml}$ lysozyme [Invitrogen], 1× protease inhibitor cocktail [Roche]). Cells were lysed by sonication and centrifuged. Clarified lysate was mixed by rotation with a 50\% Ni-NTA slurry (QIAGEN) previously equilibrated in lysis buffer and loaded in gravity flow columns (Bio-Rad). After extensive washing in wash buffer (50 $\mathrm{mM} \mathrm{NaH}_{2} \mathrm{PO}_{4}$, $300 \mathrm{mM} \mathrm{NaCl}, 20 \mathrm{mM}$ imidazole, $10 \% \mathrm{v} / \mathrm{v}$ glycerol, $\mathrm{pH} 7.4[\mathrm{NaOH}])$, bound material was serially eluted in elution buffer $\left(50 \mathrm{mM} \mathrm{NaH}_{2} \mathrm{PO}_{4}, 300 \mathrm{mM} \mathrm{NaCl}, 20 \mathrm{mM}\right.$ imidazole, 10\% v/v glycerol, $\mathrm{pH} 7.4$ $[\mathrm{NaOH}])$. Fractions were analysed by SDS-PAGE and Coomassie staining to determine purity, and pure fractions were pooled and glycerol added to a final concentration of $30 \% \mathrm{v} / \mathrm{v}$. 


\section{Recombinant Acetyl-K206 MITF}

cDNA encoding the helix-loop-helix region of the human MITF was fused by polymerase chain reaction (PCR) downstream to a Tobacco Etch Virus (TEV)-cleavable N-terminal hexa histidine affinity tag (extension: MHHHHHHSSGVDLGTENLYFQ*A, where A is the first amino acid of the MITF fragment) in presence of Herculase II fusion DNA polymerase (Agilent Technologies). An Amber codon (TAG) was introduced by PCR-based mutagenesis at K206 and the final PCR product subcloned in frame between the NcoI/XhoI sites of the pCDF-pYIT vector. Acetylation of the MITF protein at K206 was performed in vivo using amber stop codon/suppressor tRNA technology with several modifications that increased overall yields from $10 \%$ to above $70 \%$ as previously described ${ }^{81}$. In brief, the Amber K206 mutant MITF was co-expressed in TunerTM BL21 DE3 cells (Novagen, \#70622) with an orthogonal N(epsilon)-acetyl-lysyl-tRNA synthetase/tRNA(CUA) pair, leading to site specific incorporation of $\mathrm{N}$ (epsilon)-acetyl-lysine. Cells were grown at $37^{\circ} \mathrm{C}$ and at an $\mathrm{OD}_{600}$ of $\sim 0.7$ the culture was supplemented with $20 \mathrm{mM}$ nicotinamide (NAM, Sigma, \#N3376-100G) and $25 \mathrm{mM}$ acetyl-lysine (H-Lys-Ac-OH, GL Biochem Shangai Ltd, \#GLS140325). Protein expression was induced 30 min later by addition of $50 \mu \mathrm{M}$ IPTG and cells harvested by centrifugation $\left(8,700 \mathrm{x} \mathrm{g}, 15 \mathrm{~min}, 4{ }^{\circ} \mathrm{C}\right.$; Beckman Coulter Avanti J-20 XP centrifuge) after $6 \mathrm{~h}$ at $37^{\circ} \mathrm{C}$ before re-suspension in lysis buffer $[50 \mathrm{mM}$ HEPES, pH 7.5 at $20^{\circ} \mathrm{C}, 500 \mathrm{mM} \mathrm{NaCl}, 5 \%$ glycerol, $1 \mathrm{mM}$ tris(2-carboxyethyl)phosphine (TCEP) and 1:1000 (v/v) Protease Inhibitor Cocktail III (Calbiochem)]. Cells were then lysed three times at $4^{\circ} \mathrm{C}$ using a Basic Z Model Cell Disrupter (Constant Systems Ltd, UK) and DNA removed by precipitation on ice for $30 \mathrm{~min}$ with $0.15 \%(\mathrm{v} / \mathrm{v})$ of polyethyleneimine (PEI). Lysate was cleared by centrifugation $\left(16,0003 \mathrm{~g}\right.$ for $1 \mathrm{~h}$ at $4^{\circ} \mathrm{C}$ ). The supernatant was applied to a Cobalt-based IMAC resin column (Talon, GE Healthcare, $5 \mathrm{~mL}$, equilibrated with $20 \mathrm{~mL}$ lysis buffer) and the column washed once with $30 \mathrm{~mL}$ of lysis buffer, and then with $20 \mathrm{~mL}$ of lysis buffer containing $30 \mathrm{mM}$ Imidazole. Protein was next eluted using a step gradient of imidazole in lysis buffer $(50,100,150,2$ x $250 \mathrm{mM}$ imidazole in $50 \mathrm{mM}$ HEPES, pH 7.5 at $25^{\circ} \mathrm{C}, 500 \mathrm{mM} \mathrm{NaCl}$ and $5 \%$ glycerol). All fractions were collected and monitored by SDSpolyacrylamide gel electrophoresis (Bio-Rad Criterion Precast Gels, 4\%-12\% Bis-Tris, $1.0 \mathrm{~mm}$, from Bio-Rad, CA.). Clean fractions were pooled and protein was treated overnight at $4{ }^{\circ} \mathrm{C}$ with TEV protease to remove the hexa-histidine tag. Untagged protein was further purified by size exclusion chromatography on a Superdex 75 16/60 HiLoad gel filtration column (GE Healthcare Life Sciences) on an AktaPrime plus system (GE/Amersham Biosciences). Recombinant acetylated MITF eluted as single symmetrical monomeric peak. Eluted protein fractions were monitored by SDS-polyacrylamide gel electrophoresis and concentrated in gel filtration buffer (10 mM HEPES pH 7.5, $500 \mathrm{mM} \mathrm{NaCl}$ and 5\% glycerol) using Amicon Ultra (EMD Millipore) concentrators with a 3 MWCO cut-off. Protein was aliquoted into $100 \mathrm{~mL}$ fractions, flash frozen in liquid nitrogen and stored at $80^{\circ} \mathrm{C}$ until further use. Protein handling was performed on ice or in a cold room. To verify the degree of acetylation, purified acetyl MITF was subject to Electro-spray Quadrupole Time of Flight Mass Spectrometry. To this end, 
purified protein was diluted to $1 \mathrm{mg} / \mathrm{mL}$ with $0.1 \%$ formic acid and $60 \mathrm{~mL}$ injected on an Agilent 6530 QTOF(Agilent Technologies - Palo Alto, CA) mass spectrometer with a Zorbax $5 \mathrm{~mm}$ 300SB-C3 column (Agilent Technologies - Palo Alto, CA) to ascertain the correct intact mass of the acetylated protein (12.697 kDa for the untagged, monoacetylated MITF). Raw ion count data was deconvoluted using the Mass Hunter WorkStation software, Qualitative Analysis Vs B.06.00 (Agilent Technologies, Palo Alto, CA). The correct intact mass within 1 Da was confirmed for recombinant protein.

Chromatin immunoprecipitation and ChIP-Seq Cells from $30 \quad 80 \%$ confluent $15 \mathrm{~cm}$ dishes were trypsinised, collected into a $50 \mathrm{ml}$ falcon tube (Corning; Cat\# 430828), centrifuged ( $800 \times \mathrm{g}, 4 \mathrm{~min}$ ) and media aspirated in a batch of 3 dishes per each biological replicate before cross-linking in $45 \mathrm{ml}$ icecold $0.4 \%$ paraformaldehyde PBS at room temperature with rotation for 10'. Quenching was carried out by adding glycine to a final concentration of $0.2 \mathrm{M}$ and rotate for a further 10 ' and collected by centrifugation $(1500 \times \mathrm{g}, 10 \mathrm{~min})$ and snap-freeze on dry-ice. Each cell pellet was lysed in $1 \mathrm{ml}$ ChIP lysis buffer (50 mM Tris- $\mathrm{HCl}(\mathrm{pH} 8.0), 10 \mathrm{mM}$ EDTA, $10 \mathrm{mM}$ sodium butyrate, 1\% SDS, 4×PIC (Roche; Cat\#05056489001)) and pass through a 25 guage needle until there were no visible clumps before sonicated for around $12 \mathrm{~min}$ in a Covaris S220 (Peak incident $=145 \mathrm{~W}$, Duty Factor $=8 \%$, Cycle/Burst $=200$ ). Fragmentation was assessed on Bioanalyzer using Agilent High Sensitivity DNA Kit (Agilent\#5067-4626) additional sonication was carried out until 200-400 bp range was obtained. The sonicated chromatin was precleared prior to the ChIP by centrifugation at $13,000 \times \mathrm{g}, 10 \mathrm{~min}$. The supernatant for each biological replicate was pooled and transferred to a $50 \mathrm{ml}$ DNA-LoBind tubes (Eppendorf\# EP0030122232) prior to 8-fold dilution in ChIP dilution buffer (16.7 mM Tris (pH 8.0), $167 \mathrm{mM} \mathrm{NaCl}, 1.2 \mathrm{mM}$ EDTA, 1\% Triton X-100, 0.01\% SDS). $120 \mu \mathrm{g}$ of anti-HA antibody (Roche; Cat\# 11666606001) was used per biological replicate and rotated overnight. In parallel $550 \mu 1$ Dynabeads protein-G were pre-equilibrated in $1 \mathrm{ml} \mathrm{ChIP} \mathrm{dilution} \mathrm{buffer,} \mathrm{washed} \mathrm{and} \mathrm{resuspended} \mathrm{in}$ $500 \mu \mathrm{l}$ ChIP dilution buffer supplemented with $0.5 \mathrm{mg} / \mathrm{ml}$ BSA. Antibody capturing was done using blocked-Dynabeads for $1 \mathrm{~h}, 4^{\circ} \mathrm{C}$ with rotation. Washing was performed in low salt wash buffer $(20 \mathrm{mM}$ Tris- $\mathrm{HCl}$ (pH 8.0), $150 \mathrm{mM} \mathrm{NaCl}, 2$ mM EDTA, 1\% Triton X-100, 0.1\% SDS), high salt wash buffer (20 mM Tris- $\mathrm{HCl}$ (pH 8.0), $500 \mathrm{mM} \mathrm{NaCl}, 2$ mM EDTA, 1\% Triton X-100, 0.1\% SDS) and LiCl wash buffer (10 mM Tris-HCl (pH 8.0), $250 \mathrm{mM} \mathrm{LiCl,} 1 \mathrm{mM}$ EDTA, 1\% sodium deoxycholate,1\% NP-40) for 3 times in each buffer with beads transferred to a new DNA LoBind tube (Eppendorf; Cat\# Z666548) after each wash. Elution was performed in $0.2 \mathrm{ml}$ warm elution buffer (100 mM NaHCO $3,1 \% \mathrm{SDS})$. Reverse cross-linking of ChIPed-DNA was done in two-step. First, addition of $0.3 \mathrm{M} \mathrm{NaCl}$ (final concentration) and 100 unit RNAse (Life Tech\#AM2294) and incubated at $55^{\circ} \mathrm{C} 2 \mathrm{~h}$. Second, $18 \mu \mathrm{g}$ Proteinase K (Sigma\#3115828001) was added and incubated for a further $2 \mathrm{~h}$. ChIPed-DNA was purified using QIAquick PCR Purification Kit (QIAGEN; Cat\# 28106) concentration determined using Qubit dsDNA HS Assay Kit (Invitrogen; Cat\# Q32851). Library preparation and 75 bp paired-end sequencing on an Illumina HiSeq 4000 at the Wellcome Trust Centre for Human Genetics (Oxford, UK). 
Nucleic Acid methods Total RNA was extracted cells using the RNeasy mini kit (QIAGEN\#74104). cDNA was generated using QuantiTect Reverse Transcription Kit (QIAGEN\#205313). PCR was carried out using KAPA HiFi HotStart ReadyMix (KAPABiosystem\#KK2601).

Bioinformatic analysis The two technical replicates (separate flow cell) were stitched together in UNIX to generate a single fastq. Raw fastq files were fastQCed, processed and mapped to human genome build hg38 (GRCh38v23) using STARv 2.5.1b ${ }^{82}$. Mapped SAM-files were used for peak calling using the Homer package ${ }^{83}$. Input from each sample were used as control for peak calling. Annotation, intersection, gene ontology, genome ontology, bedgraph and multiwig were performed with Homer. de novo motif identification was carried out using MEME. Exact peak size was used for all the analysis rather than arbitrary extending reads from the peak summit using average fragment size in each experiment. Histogram of read density were visualised using TreeView ${ }^{84}$.

Fluorescence anisotropy assay The following fluorescein-labeled oligonucleotides were synthesized at METABION (Planegg/Steinkirchen, Germany):

Consensus : 5'- GAGATCACGTGATGAC-3'-Fluorescein

E-box : $\quad$ 5'-GAGACCACGTGTTGAC-3'-Fluorescein

M-box : $\quad$ 5'-GAGATCATGTGTTGA C -3'-Fluorescein

SR : $\quad$ 5'-GAGATCACGACTTGAC -3'-Fluorescein

These oligonucleotides were annealed with complementary unlabeled oligonucleotides through incubation at $95{ }^{\circ} \mathrm{C}$ for $5 \mathrm{~min}$, followed by a passive cooling step to room temperature. Increasing concentrations of MITF proteins were incubated with the respective dsDNA oligonucleotides at a final concentration of $1.33 \mathrm{nM}$ at $25^{\circ} \mathrm{C}$ for $5 \mathrm{~min}$ in $10 \mathrm{mM}$ Tris/ $\mathrm{HCl} \mathrm{pH} \mathrm{7.5,} 300 \mathrm{mM} \mathrm{NaCl}, 0.01 \%$ TRITON$\mathrm{X} 100$, and $0.1 \mathrm{mg} / \mathrm{mL}$ BSA. Fluorescence anisotropy was then measured using an Infinite M1000 plate reader (TECAN) using the excitation diode at $470 \mathrm{~nm}$ and detecting the emitted light at $530 \mathrm{~nm}$. Binding data were analyzed using the GraphPad Prism software. Binding profiles were fitted using a simple model assuming a stoichiometry of one MITF dimer per double stranded DNA fragment. When $\mathrm{K}_{\mathrm{D}}$ values were higher than $500 \mathrm{nM}$, the maximum specific binding value (Bmax) was estimated according to the one obtained from other variants and maintained fixed for the fitting. $K_{D}$ values reported in Fig. $3 \mathrm{~b}$ correspond to the means of three independent measurements and the $+/$ - error numbers represent the standard deviations.

\section{Single Molecule Tracking}

SMT analysis was performed in 4-well Nunc Labtek Chambers (Thermo-Fisher, Milan, Italy) on cells stably expressing doycycline-inducible HALO-tagged MITF WT or K206 mutants treated for $24 \mathrm{~h}$ with 
$20 \mathrm{ng} / \mathrm{ml}$ of doxycycline. The HALO tag was labelled using $100 \mathrm{pM}$ of the cell-permeable HaloTag ligand Janelia Fluor ${ }^{\circledR} 549$ (Janelia Farm, Ashburn, VA, USA) to each well. After 30 min incubation cells were extensively washed with PBS, incubated for $20^{\prime}$ at $37^{\circ} \mathrm{C}$ in culture medium, followed by one last wash with PBS and one in RPMI 1640. The acquisition of single molecule movies were performed according to two different regimes on a custom-built HiLo microscope, equipped with a stage temperature and $\mathrm{CO}_{2}$ controller (OkoLab, Naples, IT) - set to $37^{\circ} \mathrm{C}$ and $5 \%$ respectively -, a 561 laser (Cobolt 06-01 series, Hubner Photonics, DE) and an Hamamatsu Fusion sCMOS camera (Hamamatsu Photonics Italia SRL, Arese, IT) . To quantify diffusion coefficients and bound fraction, movies were collected using stroboscopic illumination setting the laser exposure of $5 \mathrm{~ms}$, the laser power density to approximately $0.8 \mathrm{~kW} / \mathrm{cm}^{2}$ and the acquisition frame rate to $100 \mathrm{fps}$. Movies were tracked using the ImageJ/Fiji plugin Trackmate ${ }^{85}$, and tracks were then analysed using our previously described software developed in Matlab ${ }^{50,54}$. Briefly, the tracks are used to populate the histogram of displacements (bin size $\Delta r=20 \mathrm{~nm}$ ), that are then fit with a three-component diffusion model that provides the probability of observing a displacement in the interval $\left[r-\frac{\Delta r}{2}, r+\frac{\Delta r}{2}\right]$ :

$$
p(r)=r \Delta r \sum_{i=1}^{3} \frac{F_{i}}{2 D_{i} \Delta t} \exp \left(-\frac{r^{2}}{4 D_{i} \Delta t}\right)
$$

Where $\Delta t$ is the interval between two acquisitions $(10 \mathrm{~ms})$ and $F_{i}$ is the fraction of molecules with diffusion coefficient $D_{i}$. To provide errors on the fitting parameters we applied a jacknife procedure, as described ${ }^{54}$, by performing multiple (2000) fitting iterations, each of them after dropping $20 \%$ of the data. The slowest diffusing component $\left(D_{1}=0.034 \mu \mathrm{m}^{2} / \mathrm{s}\right)$ is compatible with previously reported diffusion coefficients of chromatin bound proteins, so that $F_{1}$ represents the fraction of molecules detected as bound.

In order to measure the duration of MITF binding events (the residence times), we acquired movies at lower laser power (scaled down by a factor 20) and collected movies using long exposures (200ms exposure, frame rate $2 \mathrm{~Hz}$ ), in order to blur out diffusing molecules and selectively image immobilized ones ${ }^{56}$. These slow movies were analysed by kymograph analysis as described in ${ }^{54}$, and the duration of the immobile track segments were used to populate the complement cumulative distribution of the duration of binding events, $1-C D F$ - accounting for immobile molecules lasting longer than 5 frames $(2.5 \mathrm{~s})$. Disappearance of bound molecules can be due to either unbinding or photobleaching. To correct for photobleaching artifacts we adopted a recently described procedure ${ }^{57}$. Briefly, we collected the apparent distribution of residence times for HaloTag-H2B upon the same experimental conditions. The H2B distribution was then fit with a multi-exponential decay and the slowest component of such fit was used to provide an estimate of the photobleaching rate $\left(k_{b}=0.0316 s^{-1}\right)$. The corrected distributions of residence times show in Fig $2 \mathrm{E}$, were then obtained by dividing the uncorrected distributions for 
$\exp \left(-k_{b} t\right)$. Error bars are provided by generating multiple (1000) distributions of residence times, each after dropping $50 \%$ of the data.

\section{Data availability}

ChIP-seq dataset can be downloaded from: GSE137776 (Reviewer token: kzozeuuwnfinlst)

\section{AUTHOR CONTRIBUTIONS}

Conceptualization, CRG

Supervision, CRG, ES, MW, EEP, PF, DM

Visualization, PL, SP, AS, ZZ

Formal Analysis, PL, AL, VP, ZZ, BT

Investigation, PL, AL, HF, AS, ZZ, VP, SP, BT

Writing-Original draft, CRG, PL

Writing-review and Editing, CRG, PL, DM

Funding acquisition, CRG, ES, EEP, PF, MW, DM

\section{ACKNOWLEDGEMENTS}

The Piggybac vectors were kindly provided by Kazuhiro Murakami (RIKEN, Kobe, Japan). This work was funded by the Ludwig Institute for Cancer Research (CRG), Cancer Research UK (CRUK) grant number C38302/A12981, through a CRUK Oxford Centre Prize DPhil Studentship (HF), The Medical Research Council (PF MR/N010051/1; ZZ; EEP MC_UU_00007/9), L'Oreal-Melanoma Research Alliance 401181 (EEP), The Wellcome Trust (AS) and The Postdoc Fund of the University of Iceland (AS), The Research Fund of Iceland (ES), and a European Research Consolidator Award (ZF-MELCHEMBIO 648489) (EEP)

'This research was funded in whole, or in part, by the Wellcome Trust [Grant number]. For the purpose of Open Access, the author has applied a CC BY public copyright licence to any Author Accepted Manuscript version arising from this submission.'

\section{DECLARATION OF INTERESTS}

The authors declare no competing interests

\section{AUTHOR INFORMATION}

Correspondence and requests for materials should be sent to CRG (colin.goding@ludwig.ox.ac.uk) 


\section{REFERENCES}

1. Travnickova, J. et al. Zebrafish MITF-low melanoma subtype models reveal transcriptional subclusters and MITF-independent residual disease. Cancer Res (2019).

2. Klemm, S.L., Shipony, Z. \& Greenleaf, W.J. Chromatin accessibility and the regulatory epigenome. Nat Rev Genet 20, 207-220 (2019).

3. Zhu, F. et al. The interaction landscape between transcription factors and the nucleosome. Nature 562, 76-81 (2018).

4. Lickwar, C.R., Mueller, F., Hanlon, S.E., McNally, J.G. \& Lieb, J.D. Genome-wide protein-DNA binding dynamics suggest a molecular clutch for transcription factor function. Nature 484, 251-255 (2012).

5. Goding, C.R. \& Arnheiter, H. MITF - The first 25 years. Genes Dev 33, 983-1007 (2019).

6. Hodgkinson, C.A. et al. Mutations at the mouse microphthalmia locus are associated with defects in a gene encoding a novel basic-helix-loop-helix-zipper protein. Cell 74, 395-404 (1993).

7. Kawakami, A. \& Fisher, D.E. The master role of microphthalmia-associated transcription factor in melanocyte and melanoma biology. Lab Invest 97, 649-656 (2017).

8. Garraway, L.A. et al. Integrative genomic analyses identify MITF as a lineage survival oncogene amplified in malignant melanoma. Nature 436, 117-22 (2005).

9. Hoek, K.S. et al. In vivo switching of human melanoma cells between proliferative and invasive states. Cancer Res 68, 650-656 (2008).

10. Carreira, $\mathrm{S}$. et al. Mitf cooperates with Rb1 and activates $\mathrm{p} 21 \mathrm{Cip} 1$ expression to regulate cell cycle progression. Nature 433, 764-769 (2005).

11. Carreira, S. et al. Mitf regulation of Dial controls melanoma proliferation and invasiveness. Genes Dev 20, 3426-3439 (2006).

12. Ploper, D. et al. MITF drives endolysosomal biogenesis and potentiates Wnt signaling in melanoma cells. Proc Natl Acad Sci USA 112, E420-9 (2015).

13. Zhang, T. et al. Mitf is a master regulator of the v-ATPase, forming a control module for cellular homeostasis with v-ATPase and TORC1. J Cell Sci 128, 2938-50 (2015).

14. Cheli, Y. et al. Mitf is the key molecular switch between mouse or human melanoma initiating cells and their differentiated progeny. Oncogene 30, 2307-18 (2011).

15. Konieczkowski, D.J. et al. A melanoma cell state distinction influences sensitivity to MAPK pathway inhibitors. Cancer Discov 4, 816-27 (2014).

16. Muller, J. et al. Low MITF/AXL ratio predicts early resistance to multiple targeted drugs in melanoma. Nat Commun 5, 5712 (2014).

17. Dugo, M. et al. A melanoma subtype with intrinsic resistance to BRAF inhibition identified by receptor tyrosine kinases gene-driven classification. Oncotarget 6, 511833 (2015).

18. Falletta, P. et al. Translation reprogramming is an evolutionarily conserved driver of phenotypic plasticity and therapeutic resistance in melanoma. Genes \& Development 31, 18-33 (2017).

19. Moller, K. et al. MITF has a central role in regulating starvation-induced autophagy in melanoma. Sci Rep 9, 1055 (2019).

20. Vivas-Garcia, Y. et al. Lineage-Restricted Regulation of SCD and Fatty Acid Saturation by MITF Controls Melanoma Phenotypic Plasticity. Mol Cell 77, 120-139 (2020).

21. Louphrasitthiphol, P. et al. MITF controls the TCA cycle to modulate the melanoma hypoxia response. Pigment Cell Melanoma Res 32, 792-808 (2019). 
22. Vazquez, F. et al. PGC1alpha expression defines a subset of human melanoma tumors with increased mitochondrial capacity and resistance to oxidative stress. Cancer Cell 23, 287-301 (2013).

23. Haq, R. et al. Oncogenic BRAF regulates oxidative metabolism via PGClalpha and MITF. Cancer Cell 23, 302-315 (2013).

24. Rambow, F., Marine, J.C. \& Goding, C.R. Melanoma plasticity and phenotypic diversity: therapeutic barriers and opportunities Genes Dev 33, 1295-1318 (2019).

25. Fisher, F. et al. Transcription activation by Myc and Max: flanking sequences target activation to a subset of CACGTG motifs in vivo. EMBO J 12, 5075-5082 (1993).

26. Fisher, F. \& Goding, C.R. Single amino acid substitutions alter helix-loop-helix protein specificity for bases flanking the core CANNTG motif. EMBO J 11, 4103-4109 (1992).

27. Blackwell, T.K., Kretzner, L., Blackwood, E.M., Eisenman, R.N. \& Weintraub, H. Sequence-specific DNA binding by the c-Myc protein. Science 250, 1149-1151 (1990).

28. Solomon, D.L., Amati, B. \& Land, H. Distinct DNA binding preferences for the cMyc/Max and Max/Max dimers. Nucleic Acids Res 21, 5372-5376 (1993).

29. Hejna, M. et al. Local genomic features predict the distinct and overlapping binding patterns of the bHLH-Zip family oncoproteins MITF and MYC-MAX. Pigment Cell Melanoma Res 32, 500-509 (2018).

30. Louphrasitthiphol, P. et al. Tuning transcription factor availability through acetylationmediated genomic redistribution. Molecular Cell 79, 472-487 (2020).

31. Goding, C.R. Mitf from neural crest to melanoma: signal transduction and transcription in the melanocyte lineage. Genes Dev 14, 1712-1728 (2000).

32. Cheli, Y., Ohanna, M., Ballotti, R. \& Bertolotto, C. Fifteen-year quest for microphthalmia-associated transcription factor target genes. Pigment Cell Melanoma Res 23, 27-40 (2010).

33. Ngeow, K.C. et al. BRAF/MAPK and GSK3 signaling converges to control MITF nuclear export. Proc Natl Acad Sci USA 115, E8668-8677 (2018).

34. $\mathrm{Wu}, \mathrm{M}$. et al. c-Kit triggers dual phosphorylations, which couple activation and degradation of the essential melanocyte factor Mi. Genes Dev. 14, 301-312 (2000).

35. Price, E.R. et al. Lineage -specific signalling in melanocytes: c-Kit stimulation recruits p300/CBP to Microphthalmia. J. Biol. Chem. 273, 17983-17986 (1998).

36. Chen, Y.J., Wang, Y.N. \& Chang, W.C. ERK2-mediated C-terminal serine phosphorylation of p300 is vital to the regulation of epidermal growth factor-induced keratin 16 gene expression. J Biol Chem 282, 27215-27228 (2007).

37. Landsberg, J. et al. Melanomas resist T-cell therapy through inflammation-induced reversible dedifferentiation. Nature 490, 412-416 (2012).

38. Hugo, W. et al. Non-genomic and Immune Evolution of Melanoma Acquiring MAPKi Resistance. Cell 162, 1271-1285 (2015).

39. Hugo, W. et al. Genomic and Transcriptomic Features of Response to Anti-PD-1 Therapy in Metastatic Melanoma. Cell 165, 35-44 (2016).

40. Rambow, F. et al. Towards minimal residual disease-directed therapy in melanoma. Cell 174, 843-855 (2018).

41. Smith, M.P. et al. Inhibiting Drivers of Non-mutational Drug Tolerance Is a Salvage Strategy for Targeted Melanoma Therapy. Cancer Cell 29, 270-284 (2016).

42. Pogenberg, V. et al. Restricted leucine zipper dimerization and specificity of DNA recognition of the melanocyte master regulator MITF. Genes Dev 26, 2647-2658 (2012).

43. Sato, $\mathrm{S}$. et al. $\mathrm{CBP} / \mathrm{p} 300$ as a co-factor for the Microphthalmia transcription factor. Oncogene 14, 3083-3092 (1997). 
44. Leger, S. et al. Novel and recurrent non-truncating mutations of the MITF basic domain: genotypic and phenotypic variations in Waardenburg and Tietz syndromes. Eur J Hum Genet 20, 584-7 (2012).

45. Prince, S., Wiggins, T., Hulley, P.A. \& Kidson, S.H. Stimulation of melanogenesis by tetradecanoylphorbol 13-acetate (TPA) in mouse melanocytes and neural crest cells. Pigment Cell Res 16, 26-34 (2003).

46. Tokunaga, M., Imamoto, N. \& Sakata-Sogawa, K. Highly inclined thin illumination enables clear single-molecule imaging in cells. Nat Methods 5, 159-61 (2008).

47. Grimm, J.B. et al. A general method to improve fluorophores for live-cell and singlemolecule microscopy. Nat Methods 12, 244-50, 3 p following 250 (2015).

48. Gebhardt, J.C. et al. Single-molecule imaging of transcription factor binding to DNA in live mammalian cells. Nat Methods 10, 421-6 (2013).

49. Mazza, D., Abernathy, A., Golob, N., Morisaki, T. \& McNally, J.G. A benchmark for chromatin binding measurements in live cells. Nucleic Acids Res 40, e119 (2012).

50. Mazza, D., Ganguly, S. \& McNally, J.G. Monitoring dynamic binding of chromatin proteins in vivo by single-molecule tracking. Methods Mol Biol 1042, 117-37 (2013).

51. Kalderon, D., Roberts, B.L., Richardson, W.D. \& Smith, A.E. A short amino acid sequence able to specify nuclear location. Cell 39, 499-509 (1984).

52. Fock, V. et al. Subcellular localization and stability of MITF are modulated by the bHLH-Zip domain. Pigment Cell Melanoma Res 32, 41-54 (2018).

53. Hansen, A.S. et al. Robust model-based analysis of single-particle tracking experiments with Spot-On. Elife 7(2018).

54. Loffreda, A. et al. Live-cell p53 single-molecule binding is modulated by C-terminal acetylation and correlates with transcriptional activity. Nat Commun 8, 313 (2017).

55. Speil, J. et al. Activated STAT1 transcription factors conduct distinct saltatory movements in the cell nucleus. Biophys $J$ 101, 2592-600 (2011).

56. Chen, J. et al. Single-molecule dynamics of enhanceosome assembly in embryonic stem cells. Cell 156, 1274-1285 (2014).

57. Garcia, D.A. et al. Power-law behavior of transcription factor dynamics at the singlemolecule level implies a continuum affinity model. Nucleic Acids Res 49, 6605-6620 (2021).

58. Aksan, I. \& Goding, C.R. Targeting the microphthalmia basic helix-loop-helix-leucine zipper transcription factor to a subset of E-box elements in vitro and in vivo. Mol Cell Biol 18, 6930-6938 (1998).

59. Lowings, P., Yavuzer, U. \& Goding, C.R. Positive and negative elements regulate a melanocyte-specific promoter. Mol Cell Biol 12, 3653-62 (1992).

60. Taylor, K.L. et al. Differentiated melanocyte cell division occurs in vivo and is promoted by mutations in Mitf. Development 138, 3579-89 (2011).

61. Lister, J.A. et al. A conditional zebrafish MITF mutation reveals MITF levels are critical for melanoma promotion vs. regression in vivo. J Invest Dermatol 134, 133-40 (2014).

62. Bouras, T. et al. SIRT1 deacetylation and repression of p300 involves lysine residues 1020/1024 within the cell cycle regulatory domain 1. J Biol Chem 280, 10264-76 (2005).

63. Ohanna, M. et al. SIRT1 promotes proliferation and inhibits the senescence-like phenotype in human melanoma cells. Oncotarget 5, 2085-95 (2014).

64. Mazzocca, M., Colombo, E., Cellegari, A. \& Mazza, D. Transcription factor binding kinetics and transcritpional bursting: What do we really know? Current Opinion in Structural Biology 71, 239-248 (2021). 
65. Hipp, L. et al. Single-molecule imaging of the transcription factor SRF reveals prolonged chromatin-binding kinetics upon cell stimulation. Proc Natl Acad Sci U S A 116, 880-889 (2019).

66. Zhen, C.Y. et al. Live-cell single-molecule tracking reveals co-recognition of H3K27me3 and DNA targets polycomb Cbx7-PRC1 to chromatin. Elife 5(2016).

67. Agarwal, H., Reisser, M., Wortmann, C. \& Gebhardt, J.C.M. Direct Observation of Cell-Cycle-Dependent Interactions between CTCF and Chromatin. Biophys $J \mathbf{1 1 2}$, 2051-2055 (2017).

68. Soochit, W. et al. CTCF chromatin residence time controls three-dimensional genome organization, gene expression and DNA methylation in pluripotent cells. Nat Cell Biol 23, 881-893 (2021).

69. Corre, S. et al. Target gene specificity of USF-1 is directed via p38-mediated phosphorylation-dependent acetylation. J Biol Chem 284, 18851-62 (2009).

70. Hansen, A.S. CTCF as a boundary factor for cohesin-mediated loop extrusion: evidence for a multi-step mechanism. Nucleus 11, 132-148 (2020).

71. Boyle, S. et al. A central role for canonical PRC1 in shaping the $3 \mathrm{D}$ nuclear landscape. Genes Dev 34, 931-949 (2020).

72. Chiacchiera, F. et al. Polycomb Complex PRC1 Preserves Intestinal Stem Cell Identity by Sustaining Wnt/beta-Catenin Transcriptional Activity. Cell Stem Cell 18, 91-103 (2016).

73. Cohen, I. et al. PRC1 Fine-tunes Gene Repression and Activation to Safeguard Skin Development and Stem Cell Specification. Cell Stem Cell 22, 726-739 e7 (2018).

74. Leveille, N. \& Vermeulen, L. Polycomb complex PRC1 as gatekeeper of intestinal stem cell identity. Stem Cell Investig 3, 22 (2016).

75. Gurdon, J.B., Javed, K., Vodnala, M. \& Garrett, N. Long-term association of a transcription factor with its chromatin binding site can stabilize gene expression and cell fate commitment. Proc Natl Acad Sci U S A 117, 15075-15084 (2020).

76. Vachtenheim, J., Novotna, H. \& Ghanem, G. Transcriptional repression of the microphthalmia gene in melanoma cells correlates with the unresponsiveness of target genes to ectopic microphthalmia-associated transcription factor. J. Invest. Dermatol. 117, 1505-1511. (2001).

77. Gaggioli, C., Busca, R., Abbe, P., Ortonne, J.P. \& Ballotti, R. Microphthalmiaassociated transcription factor (MITF) is required but is not sufficient to induce the expression of melanogenic genes. Pigment Cell Res 16, 374-82 (2003).

78. Schepsky, A. et al. The microphthalmia-associated transcription factor Mitf interacts with beta-catenin to determine target gene expression. Mol. Cell. Biol. 26, 8914-8927 (2006).

79. Westerfield, M. The zebrafish book. A guide for the laboratory use of zebrafish (Danio rerio). , (Univ. of Oregon Press, Eugene, Oregon, 2000).

80. Winkler, D.F., Hilpert, K., Brandt, O. \& Hancock, R.E. Synthesis of peptide arrays using SPOT-technology and the CelluSpots-method. Methods Mol Biol 570, 157-74 (2009).

81. Savitsky, P. et al. Multivalent Histone and DNA Engagement by a PHD/BRD/PWWP Triple Reader Cassette Recruits ZMYND8 to K14ac-Rich Chromatin. Cell Rep 17, 2724-2737 (2016).

82. Dobin, A. et al. STAR: ultrafast universal RNA-seq aligner. Bioinformatics 29, 15-21 (2013).

83. Heinz, S. et al. Simple combinations of lineage-determining transcription factors prime cis-regulatory elements required for macrophage and B cell identities. Mol Cell 38, 57689 (2010). 
84. Saldanha, A.J. Java Treeview--extensible visualization of microarray data. Bioinformatics 20, 3246-3248 (2004).

85. Tinevez, J.Y. et al. TrackMate: An open and extensible platform for single-particle tracking. Methods 115, 80-90 (2017). 


\section{Fig. 1, Acetylation of MITF at K206}

a. Western blot using anti-acetyl lysine or anti-GFP antibodies of immunoprecipitated GFP-tagged MITF co-expressed with CBP, p300 or GCN5 as indicated. b. Schematic showing locations of MITF acetylation sites detected in this study. Acetylated peptides derived from Mass Spec analysis are indicated below. c. Alignment of amino acids in the basic region of related bHLH-LZ family members. Yellow highlights highly conserved residues, and the red highlight (corresponding to K206 in MITF) indicates residue within the highly conserved residues that is conserved only in the MiT subfamily. d. Depiction of the MITF-DBD-DNA co-crystal structure highlighting the K206-phosphate backbone interaction. e. Peptide array of 14 amino acid peptides containing indicated acetylated or non-acetylated residues probed with anti-acetyl K206 antibody. f. Western blot of HA-MITF expressed in cells alone

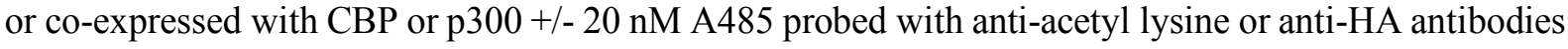
before (left-panel) or after (right panel) immunoprecipitation using anti-HA. g. Immunoprecipitation of HA-tagged MITF from IGR37 cells engineered to express doxycycline inducible p300 and HA-MITF. h. Western blot of endogenous MITF immunoprecipitated from 501mel cells using anti-MITF antibody or anti-HA as an isotype antibody control and probed with indicated antibodies. Input is shown to the left, with FT indicating the 'flow-through'. i. Immunoprecipitation of HA-tagged MITF from 501mel cells treated or not with $10 \mu \mathrm{M}$ U0126 as indicated. Inputs and immunoprecipitated protein (IP) were western blotted using the indicated antibodies with anti-ERK used as a loading control. j. Western blot of HIS-tagged MITF purified from cells treated with $\pm 200 \mathrm{nM}$ TPA for $6 \mathrm{~h}$ using nickel beads from urea dissolved cells and probed with anti-acetyl K206 or anti-MITF antibodies. Two replicates are shown, and purification of a non-his-tagged MITF ( $\Delta$ His) was used as a negative control.

\section{Fig. 2, Live-cell single-molecule tracking of HALO-tagged MITF.}

a. HALO-tagged MITF expression vectors. NLS indicates the nuclear localization sequence.

b. Single molecule tracking movie were collected at $100 \mathrm{fps}$ tracks were extracted using the FiJi/ImageJ plugin TrackMate and analysed in terms of the distribution of single-molecule displacements between consecutive frames that was then fit with a three component model (one immobile component and two diffusing components), to generate quantitative estimates for WT MITF and K206 mutants. Cmp = component. c. Quantitative estimates derived from SMT using WT and K206 HALO-tagged MITF for the fraction of molecules in each state. Error bars indicate SD. d. Quantitative estimates of the diffusion coefficients of free molecules. For WT MITF, K206R and K206Q mutants respectively $N_{\text {cells }}=28 ; 24$; $26 ; N_{\text {displacements }}=82,252 ; 102,765 ; 107,370$. Error bars indicate SD. e. Slower movies (frame rate $2 \mathrm{fps}$, laser exposure $200 \mathrm{~ms}$ ) were acquired to calculate the distribution of residence times for immobile TF molecules, following photobleaching correction using data collected on H2B-HaloTag (see methods). f. The fraction of detected bound molecules displaying a residence time longer than $100 \mathrm{~s}$ is shown. $N_{\text {cells }}=$ $43,43,45,30,35,36 ; N_{\text {bound molecules }}=5141,6193,3631,1185,2374,1225$, for WT MITF, K206R, 
K206Q, $\Delta$ basic, USF1 and p53 respectively. Error bars calculated by jacknife approach as described in the methods section.

\section{Fig. 3, Acetylation of K206 decreases binding to an M-box.}

a. DNA-binding affinity of bacterially expressed and purified MITF WT and mutant DNA-binding domains determined using fluorescence anisotropy. Representative titration curves of each fluoresceinlabeled oligonucleotide with MITF WT and mutants. The reported anisotropy values are the average of triplicate measurements, from which the base line corresponding to the anisotropy of the free fluorescent probe was subtracted. b. The calculated dissociation constants of MITF WT and mutants on oligonucleotides containing 3 different recognition sequences determined by fluorescence anisotropy.

\section{Fig. 4, K206 affects melanocyte development}

a. Amino acid sequence alignment of human and zebrafish MITF showing K206 (human) and K201 (fish) conservation. The fish mitfa coding sequence was placed under the control of the fish mitfa promoter. b. Complementation of neural crest mitfa-null nacre zebrafish using MITF WT and K201 (K206 in human MITF) mutants. c. Quantification of numbers of melanocytes in the zebrafish complementation assay. The dots in the plots represent the numbers of melanocytes in each rescued embryo with at least 1 melanocyte. $P$-values were calculated using a Two-Sample T-Test, and the line in the box plots indicates the mean.

\section{Fig. 5, K206 controls MITF genome-wide distribution.}

a. Western blot of 501mel melanoma cell lines stably expressing HA-tagged doxycycline-inducible MITF WT and K206 mutants after induction with indicated concentrations of doxycycline.b. Heatmap visualization of MITF WT and K206 mutants read density derived from HA-MITF ChIP-seq using indicated concentrations of doxycycline. Input controls are shown to the right. c. UCSC genome-browser screenshots of ChIP-seq profiles of HA-MITF WT and mutants at different concentrations of doxycycline as indicated. d. ChIP-seq peak scores for a set of lysosomal or pigmentation genes as indicated for MITF WT and mutants induced using $20 \mathrm{ng}$ doxycycline. e. Relative ratio of ChIP peaks with indicated motifs for MITF WT and mutants expressed at 0,20 and $100 \mathrm{ng}$ doxycycline. f. Consensus motifs detected beneath the ChIP peaks for MITF WT and mutants. g. Scatter plots comparing ChIP-seq peak score versus moving average of relative motif incidence (100 peaks window) for MITF WT and mutants. To aid visual comparison the data points were fitted with smoothed line using generalized additive models. The $95 \%$ confidence interval is shown in grey shade around the fitted smoothed line. 
Supplementary Fig. S1, Mass Spec. analysis of immunoprecipitated MITF ectopically expressed with p300 highlighting acetylated lysines (top) and MS/MS spectra showing b- and y-ion coverage of the Acetyl K206 peptides (bottom).

Supplementary Fig. S2, Mass Spec. spectra of bacterially expressed and purified MITF DNA binding domain showing non acetyl MITF (upper panel) and acetyl-K206 (middle panel) and the mass shift between the two (lower panel).

Supplementary Fig. S3, a. Western blot of doxycycline inducible HA-WT and mutant MITF at 0, 10 and $20 \mathrm{ng}$ doxycycline probed with anti-MITF antibody. b. Comparison of MITF WT and mutant ChIPseq peak scores for each replicate at 0,20 and $100 \mathrm{ng}$ doxycycline. c. Numbers of peaks called for each replicate ChIP-seq for MITF WT at different doxycycline concentrations. d. UCSC genome-browser screenshots of ChIP-seq profiles of HA-MITF WT and mutants at different concentrations of doxycycline as indicated. 
a

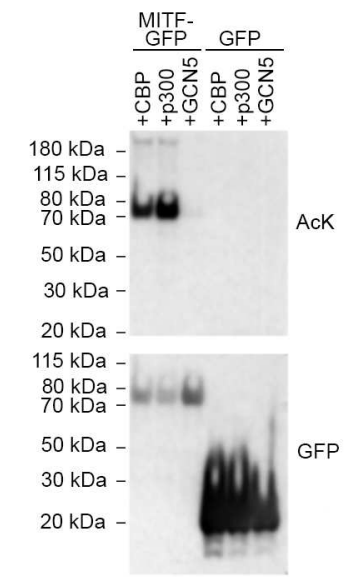

d

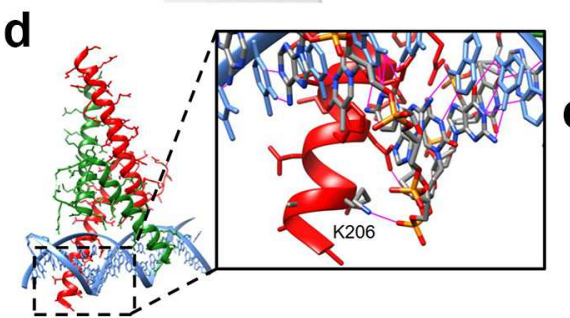

f

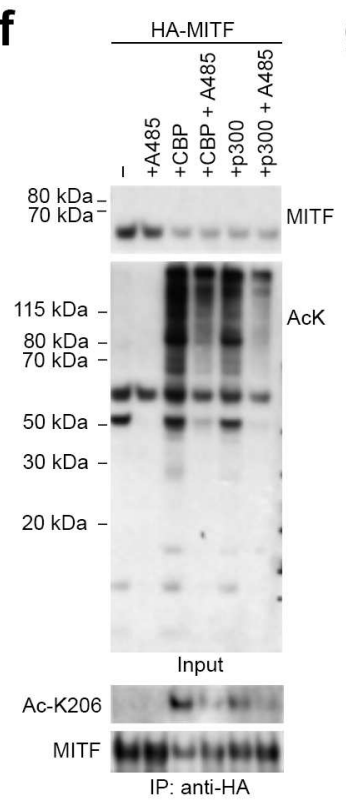

b

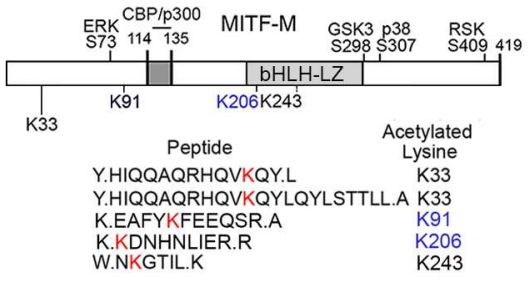

C

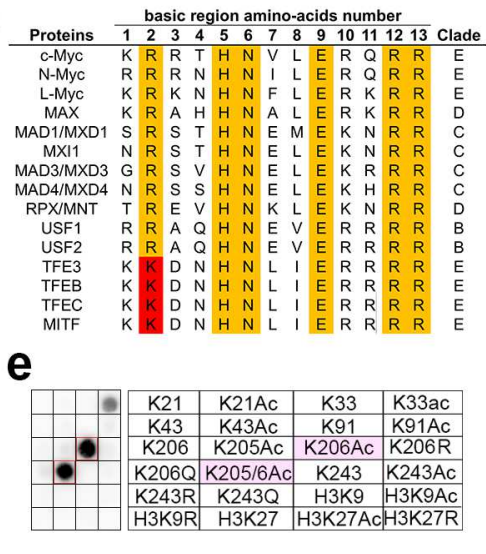

g
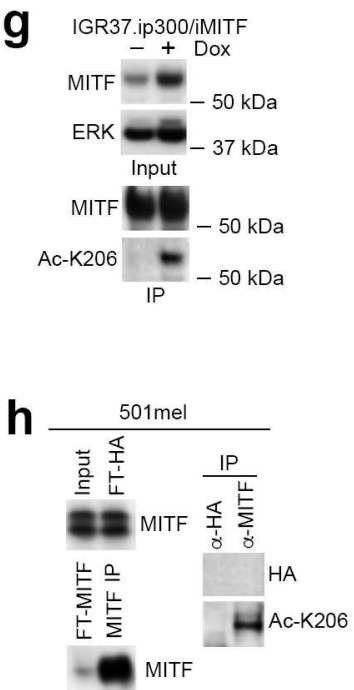

$$
\begin{aligned}
& \text { i. 501mel.iHA-MITF } \\
& \text { MITF } \overline{0}-50 \mathrm{kDa} \\
& \text { p-ERK }-37 \mathrm{kDa} \\
& \text { ERK }-37 \mathrm{kDa} \\
& \text { Input } \\
& \text { MITF } 1-50 \mathrm{kDa} \\
& \text { Ac-K206 } \begin{array}{l}
-50 \mathrm{kDa} \\
-50 \mathrm{kDa}
\end{array} \\
& \text { j } \\
& T P A \frac{R 1}{-}+\frac{R 2}{-2}+\frac{D}{7} \\
& \text { பய山比 MITF } \\
& \text { 可HBG AC-K206 } \\
& \text { EHAH Merged }
\end{aligned}
$$


a

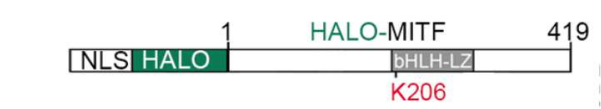

b
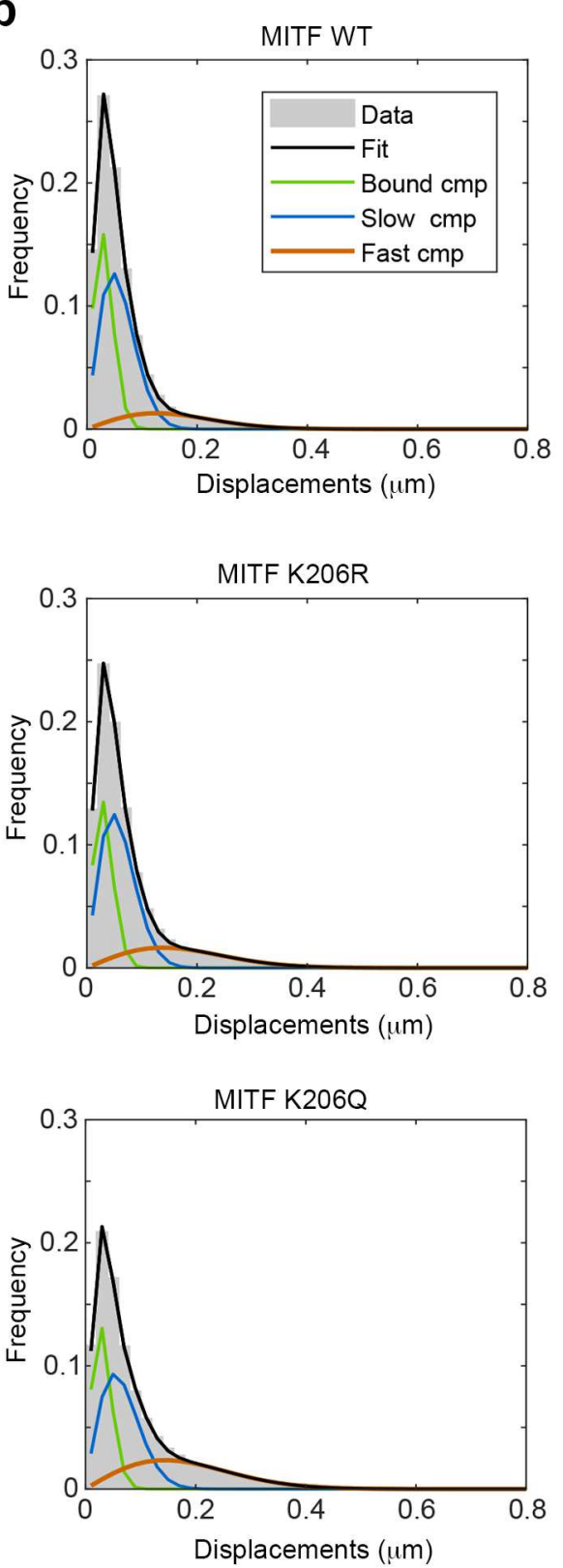

C Fraction of molecules
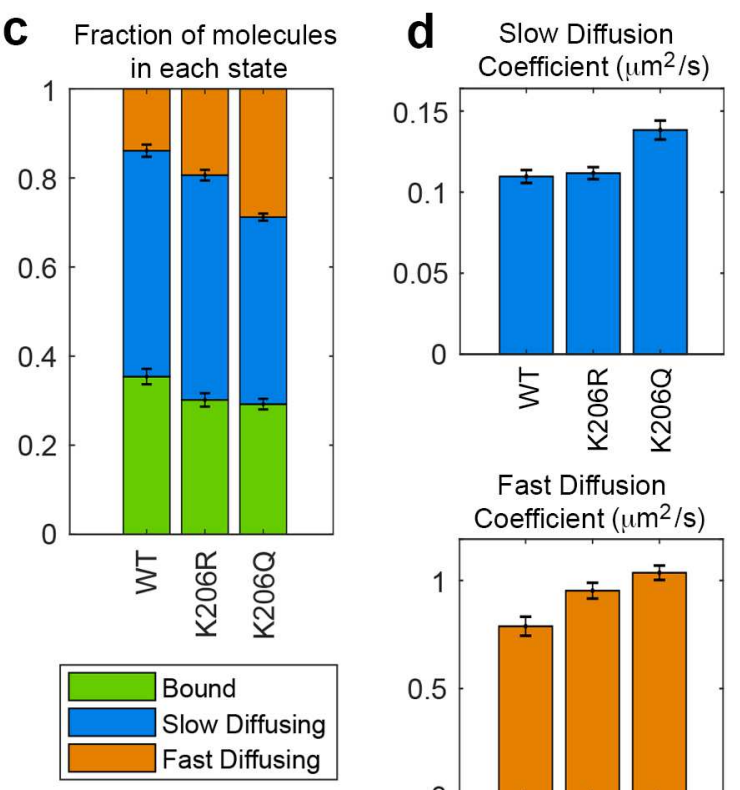

Fast Diffusion

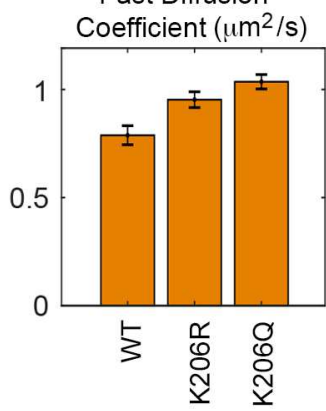

e

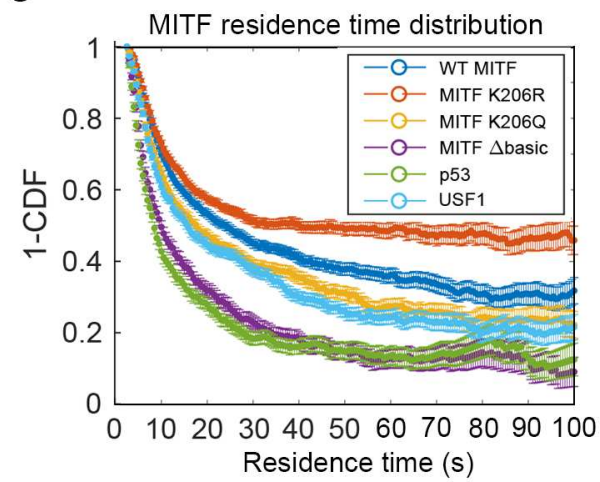

f

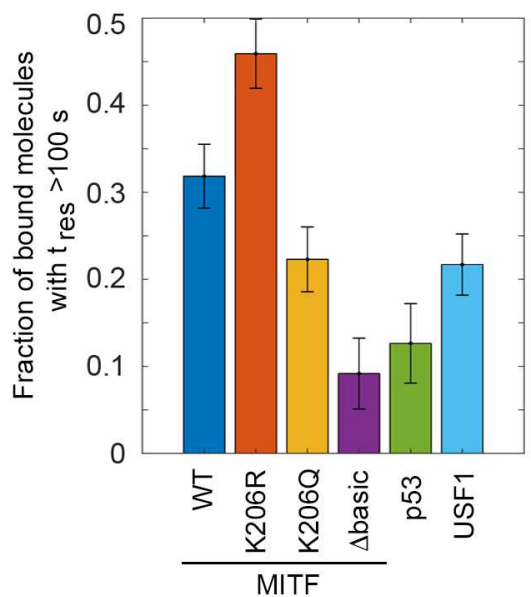


a

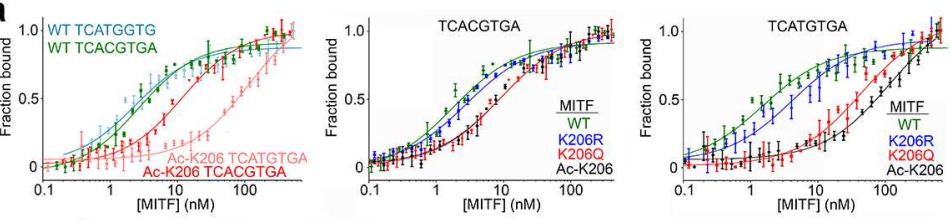

b

\begin{tabular}{|c|c|c|c|}
\hline & CLEAR box & TYR enhancer & M-box \\
\hline & TCACTGTA & TCATGTGA & \\
\hline MITF WT & $4.5 \pm 1.6$ & $3.3 \pm 1.4$ & $53 \pm 17$ \\
\hline MITF K206R & $4.6 \pm 2.3$ & $4.3 \pm 1.9$ & $21 \pm 4$ \\
\hline MITF AC-K206 & $15 \pm 4$ & $82 \pm 27$ & $300 \pm 50$ \\
\hline MITF K206Q & $11 \pm 5$ & $41 \pm 8$ & $360 \pm 100$ \\
\hline
\end{tabular}

Louphrasitthiphol et al

Figure 3 


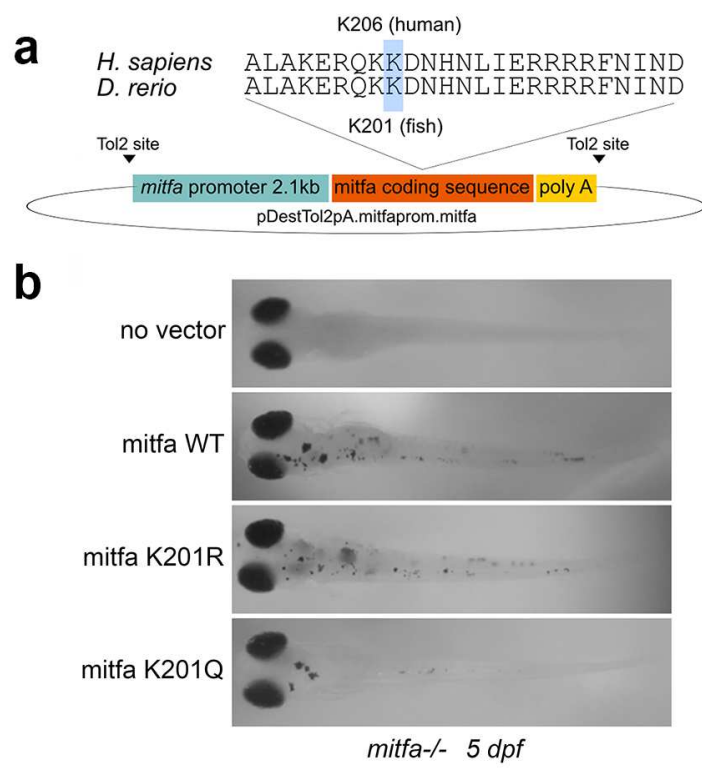

C

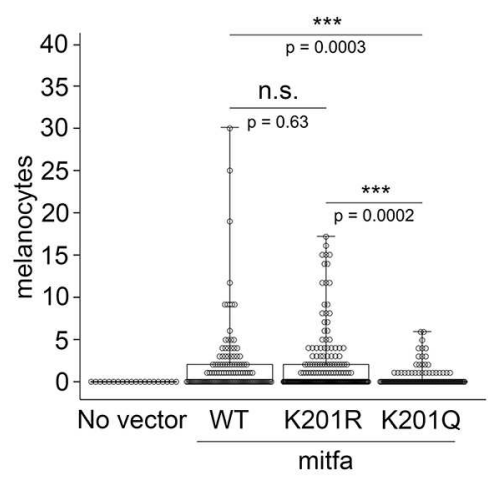

Louphrasitthiphol et al Figure 4 
a

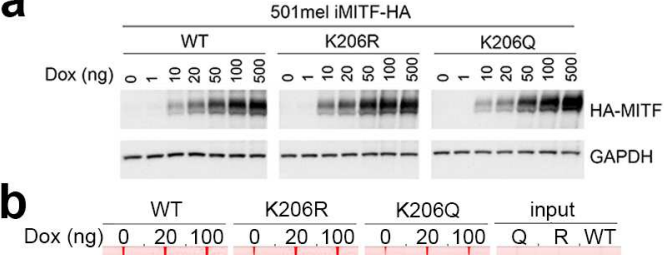

d

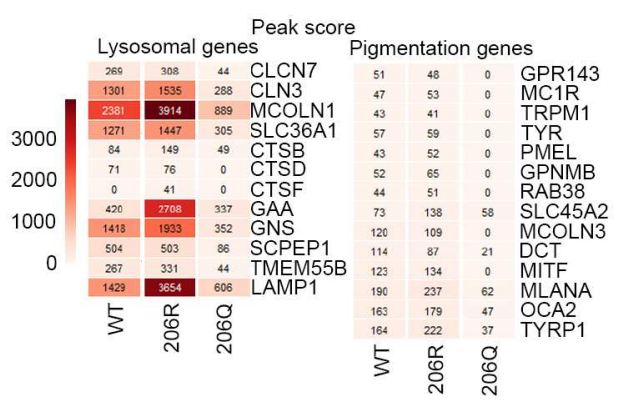

C
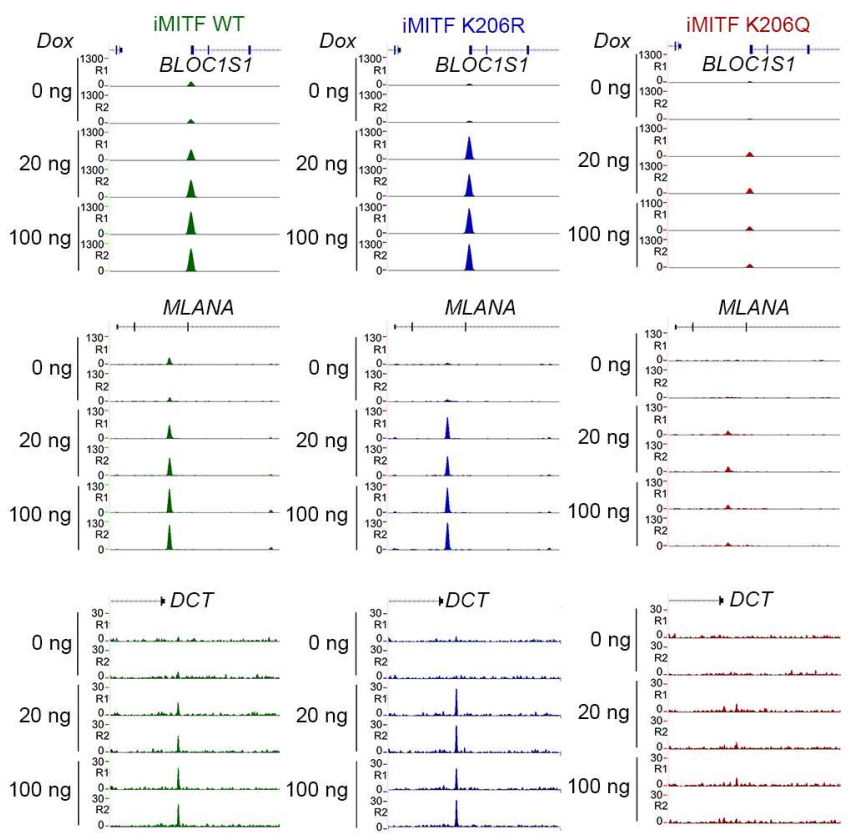

g

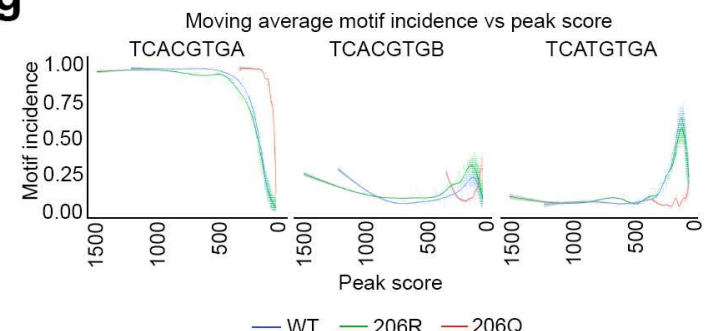

$$
\text { -WT }-206 \mathrm{R}-206 \mathrm{Q}
$$

e

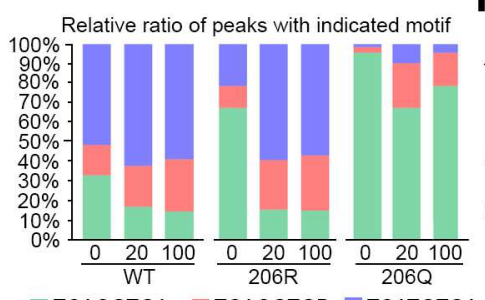

\section{$f$}

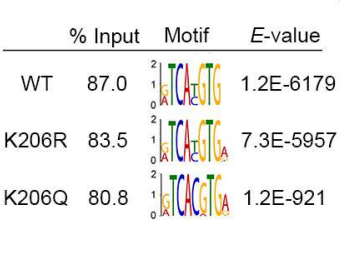

Louphrasitthiphol et al

Figure 5 


\section{Supplementary Files}

This is a list of supplementary files associated with this preprint. Click to download.

- GodingRepSum.pdf

- Supplementarydata.Combined.pdf 